\title{
Janus particles: recent advances in the biomedical applications
}

This article was published in the following Dove Press journal:

International Journal of Nanomedicine

Tu C Le'

Jiali Zhai ${ }^{2}$

Wei-Hsun Chiu ${ }^{3}$

Phong A Tran ${ }^{3,4}$

Nhiem Tran ${ }^{2}$

'School of Engineering, RMIT University, Melbourne, VIC 300I, Australia; ${ }^{2}$ School of Science, RMIT University, Melbourne, VIC 300I, Australia; ${ }^{3}$ School of

Chemistry, Physics and Mechanical Engineering, Science and Engineering

Faculty, Queensland University of Technology, Brisbane, QLD 4000,

Australia; ${ }^{4}$ Interface Science and Materials Engineering group, School of Chemistry, Physics and Mechanical Engineering, Queensland University of Technology, Brisbane, QLD 4000,

Australia
Correspondence: Nhiem Tran

School of Science, RMIT University, 124 La

Trobe Street, Melbourne VIC 3000 ,

Australia

Tel +61 399252131

Email Nhiem.tran@rmit.edu.au

\begin{abstract}
Janus particles, which are named after the two-faced Roman god Janus, have two distinct sides with different surface features, structures, and compositions. This asymmetric structure enables the combination of different or even incompatible physical, chemical, and mechanical properties within a single particle. Much effort has been focused on the preparation of Janus particles with high homogeneity, tunable size and shape, combined functionalities, and scalability. With their unique features, Janus particles have attracted attention in a wide range of applications such as in optics, catalysis, and biomedicine. As a biomedical device, Janus particles offer opportunities to incorporate therapeutics, imaging, or sensing modalities in independent compartments of a single particle in a spatially controlled manner. This may result in synergistic actions of combined therapies and multi-level targeting not possible in isotropic systems. In this review, we summarize the latest advances in employing Janus particles as therapeutic delivery carriers, in vivo imaging probes, and biosensors. Challenges and future opportunities for these particles will also be discussed.
\end{abstract}

Keywords: Janus particles, therapeutics, theranostics, imaging, sensing

\section{Introduction}

The application of nanotechnology in the biomedical field has been experiencing a tremendous development and growth over the past few decades. ${ }^{1-3}$ Nanomaterials with tunable and controllable structures at the nanometer scale offer multiple physical or chemical properties that are suitable for different functionalities and therefore will revolutionize current regime of drug delivery, treatment, and diagnosis. Regarding the application of drug delivery using nanoparticles (NPs) or nanomedicines, a vast body of research has shown benefits including improvement in delivery of hydrophobic or macromolecule-based drugs, controlled/slowed release and specific targeting to the disease sites, all leading to better bioavailability and reduced side effects. ${ }^{4-6}$ Application of NPs in imaging and diagnostics also provides benefits in precision delivery of imaging agents, multimodal imaging, and combination of therapeutics and diagnostics (theranostics). ${ }^{7,8}$ Driven by the diverse range of potentials, a variety of NP systems such as liposomes, ${ }^{9}$ polymeric NPs, ${ }^{10}$ metallic NPs, ${ }^{11}$ and self-assembled lipid NPs ${ }^{12}$ have been exploited in the biomedical research field, with some successful nanomedicine products already in the market.

Nevertheless, there is still great need for advanced NP systems to overcome the challenges of personalized treatment and diagnosis. Janus particles, among many NPs systems, have received considerable amount of interest recently as the nextgeneration "smart" nanomaterials due to their complexity and flexibility in structures and properties with a wide range of potential applications in drug delivery, imaging, 
bio-sensing, and theranostics. ${ }^{13-15}$ Janus particles, named after the two-faced roman god-Janus, are used to describe a unique class of particles that have two distinct sides that can be anisotropic in composition and surface features. More attractively, Janus NPs have tunable and controllable anisotropic structures, which allow customization of physicochemical properties down to the nanoscale. These Janus NPs often combine incompatible and complementary physical and chemical properties such as polarity, amphiphilicity, charge, optical, and magnetic properties in one single particle, making it possible to realize diverse and synergistic functionalities.

C. Casagrande and M. Veyssié in 1989 reported the first Janus "beads" that offered amphiphilicity within a single solid particle of diameters in the range of $50-90 \mu \mathrm{m} .^{16}$ These Janus "beads" were prepared from glass spheres with one side having a cellulose (hydrophilic) varnish and the other side treated by octadecyltrichlorosilane (hydrophobic). They then found when dispersed at oil-water interfaces, these Janus "beads" were always symmetrically positioned at the interface with the hydrophobic half immersed in the oil side and the hydrophilic half in water. Following these first experiments, the concept of using Janus to describe the class of NPs that have two distinct sides was brought to the attention of scientific community by De Gennes's Nobel lecture entitled "Soft Matter" in 1991. ${ }^{17}$ Initially the types of Janus NPs in terms of geometry, composition, and size were limited due to the difficulty of synthesis methods. Nevertheless, driven by diverse potential applications of Janus NPs, there has been a tremendous amount of research with a focus on developing novel fabrication methods. Several synthesis methods have been developed including surface coating, layer-by-layer self-assembly, polymerization, Pickering emulsion, and electrified jetting over the past two decades. ${ }^{18,19}$ This has led to the generation of various kinds of Janus NPs made from a range of materials including polymer-polymer, polymer-metal, ${ }^{20}$ metal-metal, ${ }^{21}$ lipidlipid, ${ }^{22}$ and lipid-polymer. ${ }^{23}$ Protein-DNA-based Janus NP have also been reported very recently. ${ }^{24}$ In terms of particle shape, Janus NPs can adopt spherical, ellipsoidal, cylindrical, or disk-like shapes. ${ }^{19}$

The development of simple synthesis routes has enabled a trend of research activities shifting towards understanding the biomedical applications of Janus NPs. Compared to NPs with a uniform composition or surface, Janus NPs have been shown to have advantages in the applications of dual drug release, combined therapy such as chemo-photothermal therapy, multimodal imaging, and cellular targeting because of the combination of at least two distinct materials and properties in a single particle. Moreover, the asymmetric structures of Janus NPs offer compartmentalization of materials with opposite or complementary properties, each imparting a different functionality. For example, Janus NP can be designed as such that one compartment of the Janus NP houses a hydrophilic drug molecule whilst the second compartment encapsulates a hydrophobic agent, enabling dual release or combined therapy. ${ }^{23}$ The problem of non-Janus particles with uniform composition, however, is often the exclusion of one drug to the other or limited encapsulation capacity within one compartment. Another advantage is the ability of real-time monitoring of sequential release of the multiple drugs or imaging agents loaded in Janus NPs, which is again unachievable in uniform NPs. ${ }^{25}$ Many other complementary functions can be combined into Janus NPs such as selective surface modification for targeting, ${ }^{26}$ biosensing, multimodal imaging, or theranostics. ${ }^{27}$

The methods to prepare various Janus particles have been reviewed and discussed previously (Figure 1). ${ }^{18,20,22,28-32}$ However, considering the rapidly growing number of work on the biomedical applications of Janus particles in the last few years, a critical review on this topic is urgently needed. The focus of this review is to summarize and discuss in detail the recent development of Janus NPs for therapeutics, in vivo imaging, theranostics, and biosensing. Synthesis methods for specific and relevant Janus particles will be discussed on a case-by-case basis.

\section{Biomedical applications of Janus particles}

This section will provide some recent developments of Janus NPs for biomedical applications. The performance of nanoparticle-based medical devices is affected by many different parameters including surface chemistry, size, shape, mechanical properties, charge, and porosity. Much effort has been made to adjust these properties to obtain desirable in vivo behaviors such as prolonged circulation, elevated tumor accumulation, improved cancer cell uptake, evasion of multiple biological barriers, and controlled drug release in the case of anti-cancer nanomedicine. The main challenge for these activities is that the parameters are interdependent, meaning that an improvement in one property may result in an undesirable change in another. Janus NPs provide advantages compared to uniform NPs due to their compartmentalised structures with multiple domains of independent properties. 


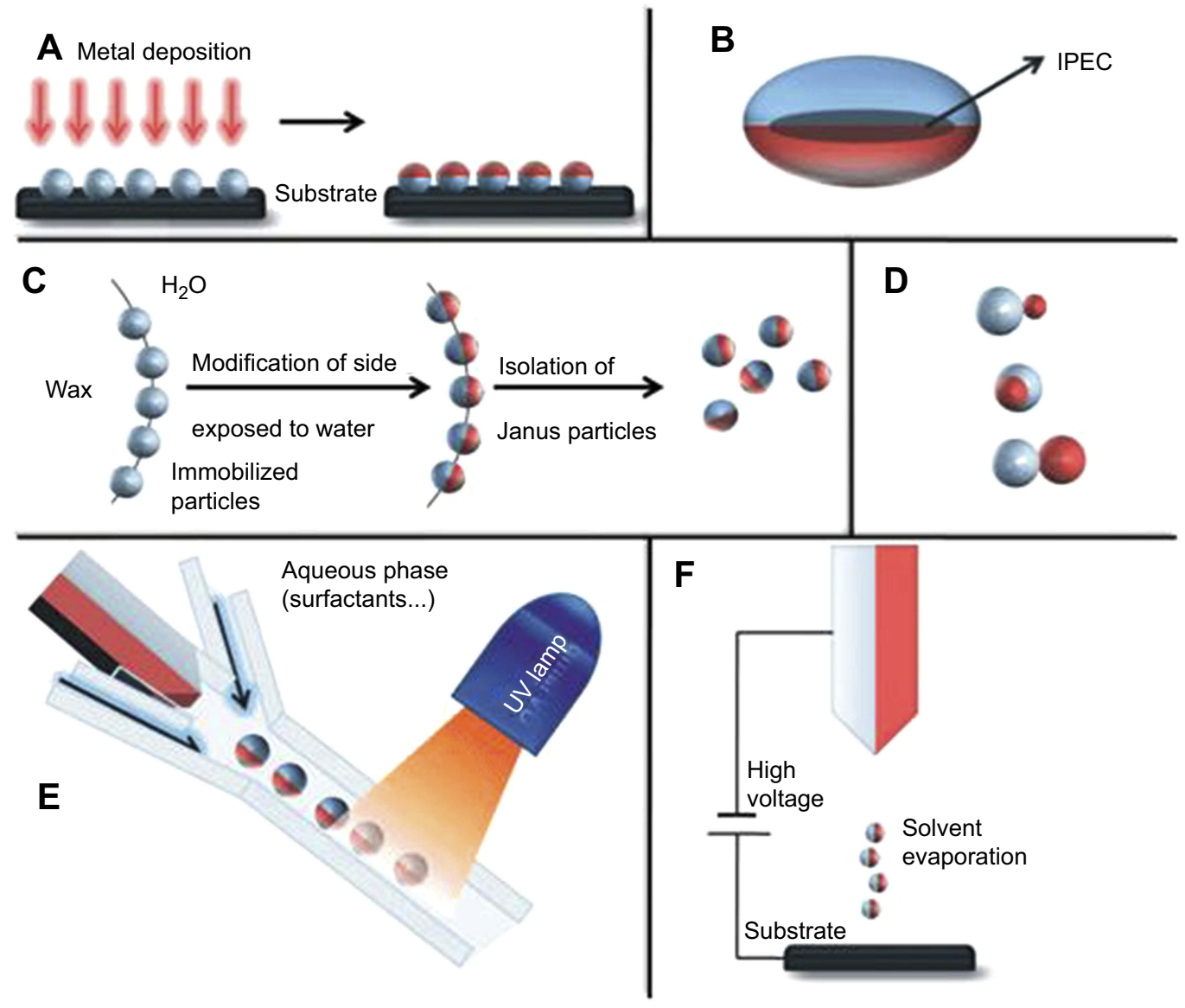

Figure I Overview of approaches towards the preparation of Janus particles. (A) Classical two-dimensional technique involving shading of one particle side after their immobilization. (B) Ellipsoidal complex core coacervate micelle with an interpolyelectrolyte complex core (IPEC). (C) Pickering emulsion route. (D) Janus particles with two inorganic compartments, snowman-, acorn-, dumbbell-like nanoparticles (top to bottom). (E) Microfluidic photopolymerization system. (F) Electrospinning using a bi-phasic nozzle. Reprinted with permission from the Walther A, Müller AH. Janus particles. Soft Matter. 2008;4(4):663-668. Copyright @ 2008 Royal Society of Chemistry. ${ }^{28}$

This allows for the incorporation of multiple functionalities in a spatially controlled manner. As a biomedical device, these unique properties may provide synergistic actions of combined therapies and multi-level targeting not possible in isotropic systems. For example, multiple materials or materials combinations can be included in a single particle to provide controlled release of multiple drugs with independent release kinetics. There are many different types of Janus NPs that maybe suitable for biomedical applications. We organize these particles into three different categories based on their materials: (1) polymeric and organic based Janus particles; (2) inorganic Janus particles; and (3) polymericinorganic composite Janus particles.

\section{Janus particles for therapeutics} Polymeric and organic-based Janus particles for therapeutics

Early development of polymer-polymer Janus particles has focused on the ability to encapsulate two different drugs in each compartment of a particle. Hwang and Lahann demonstrated the synthesis of Janus particles with differentially degradable compartments by electrohydrodynamic cojetting and crosslinking. ${ }^{33}$ These sub-micron particles are composed of poly(ethylene oxide) (PEO) and poly(acrylamide-co-acrylic acid) in one hemisphere and a crosslinked copolymer of dextran and poly(acrylamide-co-acrylic acid) segments in the second compartment (Figure 2A). As a result, the two compartments were stable at $\mathrm{pH} 3.0$ but showed different degradation rates at $\mathrm{pH} 7.4$ with the PEO containing compartment completely degraded after 5 days. Using fluorescein isothiocyanate-conjugated PEO (FITCPEO) as a model drug, the authors showed a $\mathrm{pH}$-dependent differential release rate from the Janus particles, making them promising candidates for staged drug delivery vehicles. However, these particles were not capable of carrying payloads with disparate solubility. Additionally, although this synthesis method can produce high-quality particles, it cannot achieve smaller nanoscale particles and is difficult to 

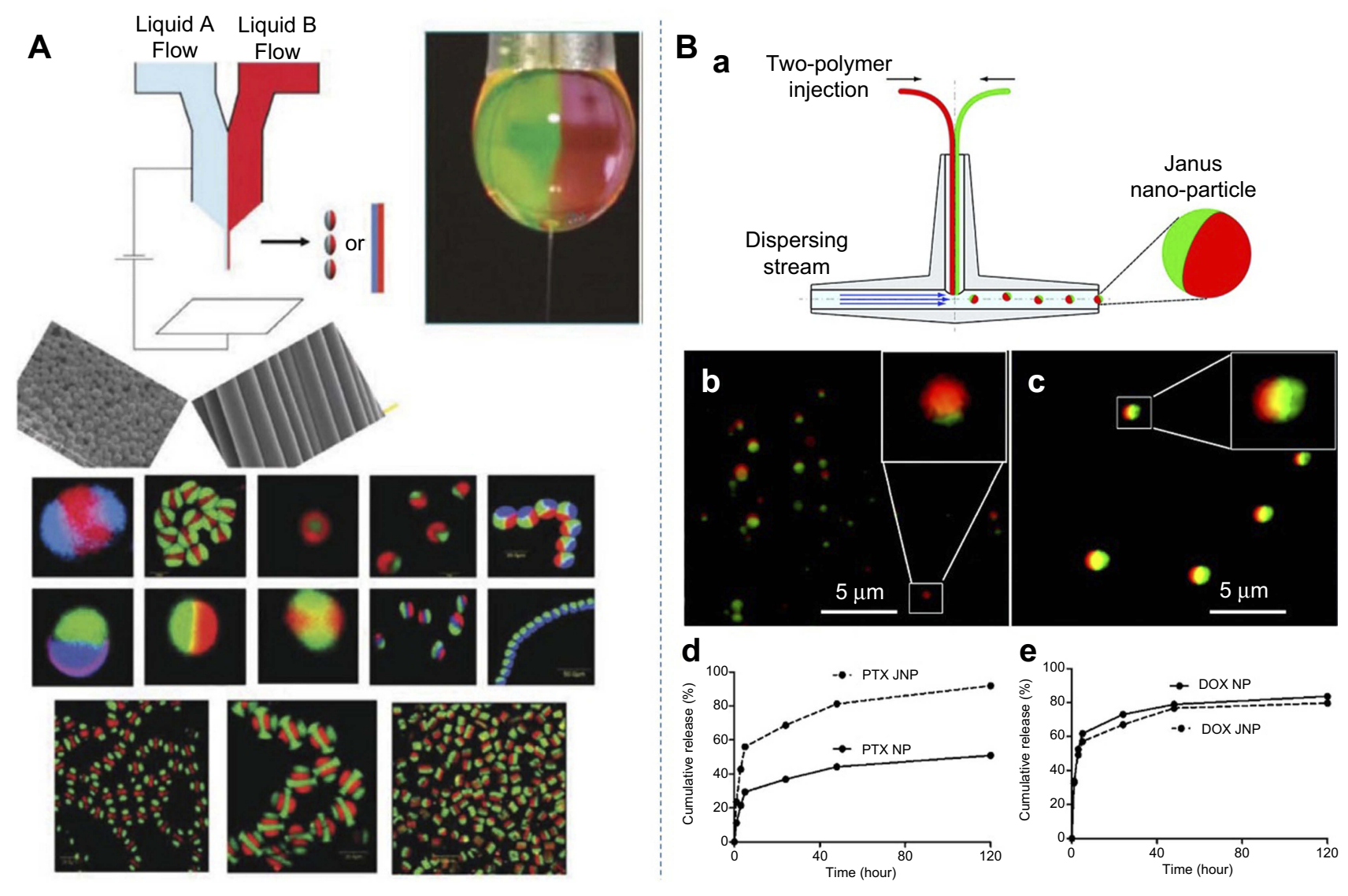

Figure 2 (A) Schematics of the electrohydrodynamic (EHD) co-jetting method developed by Lahann et al. Depending on the preparation parameters such as flow rates, polymer concentrations, potential difference, either particles (electrospraying) or fibers (electrospinning) can be obtained from the breaking of the Taylor cone (top right). Some examples showing clear asymmetry or heterogeneous nature of the obtained particles or fibers. ${ }^{37}$ (B): (a) Schematic of the modified fluidic nanoprecipitation system (FNPS); (b) Janus polymer particles loaded with two hydrophobic dyes, Nile red (red) and Rh-6G (green); (c) Janus particles loaded with Nile red and the hydrophilic dye FITC-dextran (green); (d, e) cumulative release of PTX and DOX from Janus NPs (JNP) and monophasic particles (NP). Reprinted with permission from Xie H, She Z-G, Wang S, Sharma G, Smith JW. One-step fabricationof polymeric Janus nanoparticles for drug delivery. Langmuir. 20I2;28(9):4459-4463. Copyright 2012 American Chemical Society. ${ }^{34}$

scale up. Xie et al were the first to report Janus polymeric NPs capable of carrying a hydrophobic drug (paclitaxel PTX) and a hydrophilic drug (doxorubicin hydrochloride DOX) in a single particle. ${ }^{34}$ The Janus NPs were prepared by using a fluidic nanoprecipitation system with the two compartments consisting of different types of poly-(lactic-coglycolic acid) (PLGA) (Figure 2B a, b, c). Systemic delivery of the two selected drugs is problematic and potentially can be improved by encapsulating them into particles. For example, PTX is very poorly soluble and the solvent that commonly used to deliver it, Cremophor EL, is known for causing side effects limiting its efficacy while DOX elicits cardiomyopathy. ${ }^{35,36}$ The results of this study showed that both PTX and DOX were encapsulated and released from the Janus NPs. Compared to monophasic particles, more PTX was released from Janus particles in the burst phase. In contrast, DOX was released from Janus particles at a rate similar to that from monophasic particles (Figure 2B d, e).
More recently, Dehghani et al reported the production of polymeric Janus particles that provide dual release of DOX as an anti-cancer drug and ibuprofen as an anti-inflammatory drug. ${ }^{38}$ The particles with dumbbell-like or snowman-like morphologies consisted of poly(2-hydroxyethyl methacrylate) (PHEMA) and poly(2-dimethylaminoethyl methacrylate) (PDAMEMA) and were prepared by combination of distillation precipitation polymerization and seeded emulsion polymerization. Since PHEMA is a thermoresponsive polymer and PDMAEMA is a pH-sensitive polyelectrolyte, these Janus particles possess an interesting duality responsive behavior. The drug release study demonstrated that DOX was released more rapidly than ibuprofen at both $\mathrm{pH}=7.4$ and $\mathrm{pH}=5.3$. Additionally, DOX showed higher cumulative release at lower $\mathrm{pH}$ value due to higher solubility of DOX in low-pH media whereas ibuprofen showed higher cumulative release at $\mathrm{pH}=7.4$. This release behavior cannot be achieved by coreshell type nanoparticles. 
Romanski et al developed an emulsion solvent evaporation method and were able to formulate both polymer-polymer and polymer-lipid anisotropic particles (Figure 3A). ${ }^{39}$ Three drug systems tested were griseofulvin (poorly water soluble), omeprazole (poorly water soluble), and acetaminophen (water soluble). They showed that the encapsulation of drugs did not affect the anisotropic structure of polymer-lipid particles, but it greatly affected the phase separation of polymer-polymer particles, leading to the loss of the Janus characteristic and the appearance of pox-like polymeric particles. ${ }^{39}$ Although all aforementioned studies on polymeric Janus NPs demonstrated the ability to encapsulate several drugs with different solubility, they have not been tested in vitro or in vivo, and this should be a priority in order to realize their biomedical applications. So far, the only study that investigated the in vivo behavior of polymer-polymer Janus particles that we found was performed by Rahmani et $\mathrm{al}^{40}$ This study provided interesting information regarding the biodistribution of PLGA@PLGA Janus NPs in a nondisease mouse model. The authors found that the circulation of these NPs persisted over $24 \mathrm{hrs,} \mathrm{suggesting} \mathrm{a} \mathrm{potential} \mathrm{use}$ for drug delivery applications. It would be therefore interesting to see the biodistribution of these NPs in disease models.
Following the successful formulation of polymer-lipid Janus NPs, ${ }^{39}$ they were loaded with anticancer drugs, curcumin (CUR) and DOX, for lung cancer treatment by inhalation (Figure 3B-D). ${ }^{23}$ The combination of these anticancer drugs, which had different mechanisms of action and solubility, into Janus NPs appeared to provide synergistic cytotoxic and genotoxic effects against A549 lung cancer cells in vitro. To study these NPs efficacy in vivo, an orthotopic murine model in which A549 human lung cancer cells were injected into the lung of nude mice was used. The tumors were treated by inhalation of various NP formulations and the highest anti-tumor effect was observed after inhalation of NPs simultaneously loaded with both CUR and DOX (Figure 3E-G). Compared to free DOX, NPs with DOX, and NPs with CUR, the semi-weekly treatment for 4 weeks with CUR and DOX-loaded NPs completely inhibited the growth of lung tumors. Additionally, the empty Janus NPs were found to be non-toxic to the mice. It should be pointed out that the benefit of co-encapsulating CUR and DOX in Janus particles would have been more obvious if the NP treatment was compared to a combined regime of free DOX and free CUR.

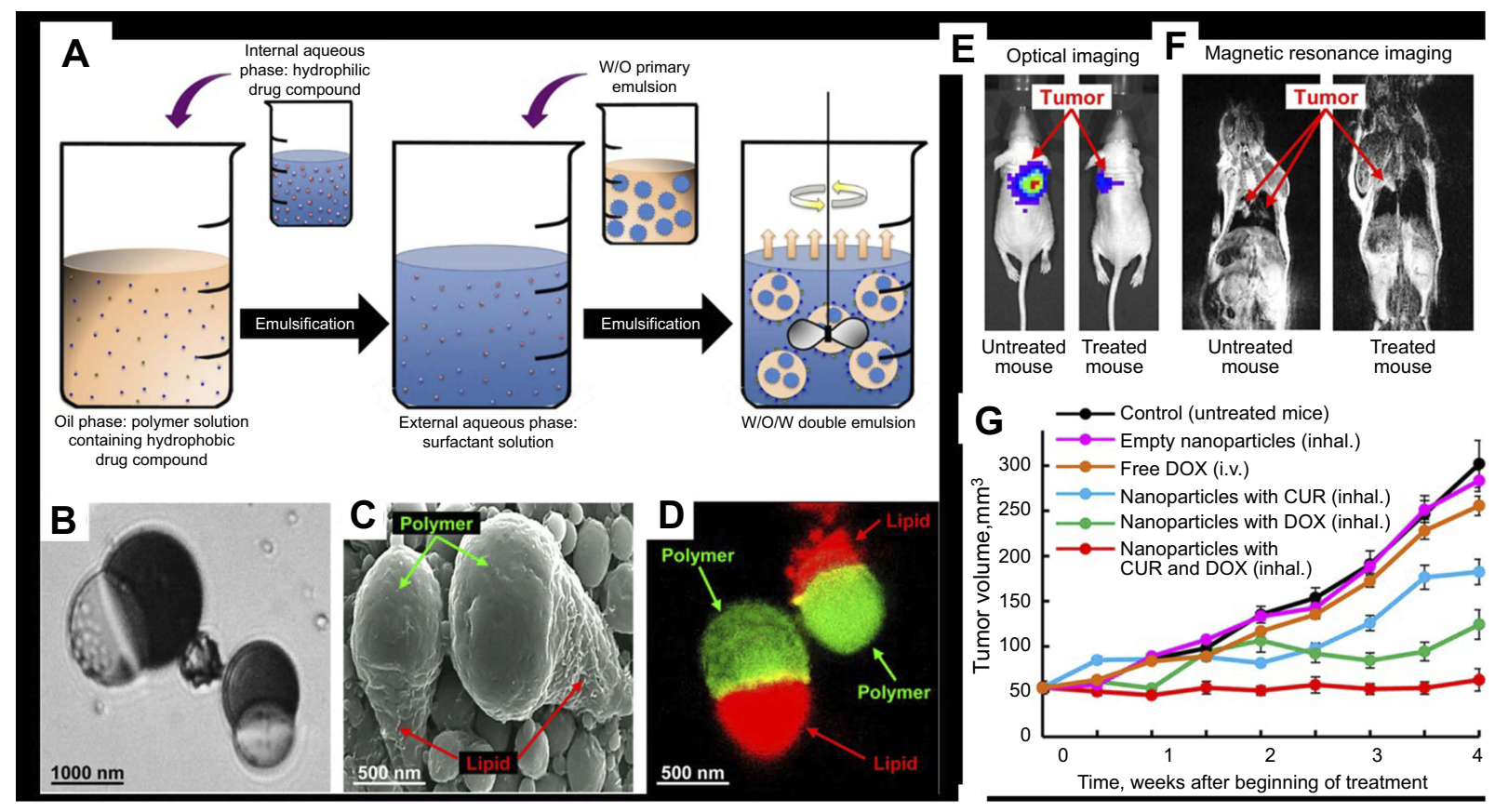

Figure 3 (A) Schematic of the formation process of PLGA@Precirol polymer-lipid Janus particles via an internal phase separation induced by solvent evaporation. (B-D) Representative optical, scanning electron microscopy, and fluorescent images of the polymer-lipid Janus NPs. (E-G) Suppression of lung tumor growth in mice inhaled with polymer-lipid Janus NPs containing CUR and DOX. Representative optical (E) and magnetic resonance (F) images 4 weeks after the injection of A549 human lung cancer cells into the lung of mice. (G) Changes in lung tumor volume after beginning of treatment with Empty NPs, free DOX, NPs with CUR, NPs with DOX, and NPs with CUR and DOX. Adapted with permission from Garbuzenko OB, Winkler J, Tomassone MS, Minko T. Biodegradable Janus nanoparticles for local pulmonary delivery of hydrophilic and hydrophobic molecules to the lungs. Langmuir. 2014;30(43):I294I-12949 (https://doi.org/I0.1021/la502I44z). ${ }^{23}$ Copyright (C 2014 American Chemical Society. 
Beside the polymer-polymer and polymer-lipid Janus NPs listed earlier, there have not been many other studies reporting on organic Janus NPs. Recently, the existence of Janus lipid NPs consisting of two lyotropic liquid crystalline compartments has been confirmed. Using a high throughput screening approach, Tran et al investigated ternary lipid nanoparticulate systems at the boundary of two bicontinuous cubic phases or a cubic phase and a hexagonal phase..$^{22,41,42}$ As a result, they provided cryogenic TEM images of primitive cubic@diamond cubic and diamond cubic@hexagonal Janus lipid NPs. These non-lamellar lipid NPs have received increasing attraction as nanocarriers for drugs, ${ }^{4,12,43,44}$ genes, ${ }^{45,46}$ and imaging contrast agents ${ }^{7}$ due to their unique self-assembled nanostructures, which control the drug release rate, encapsulation capacity of both hydrophobic and hydrophilic compounds, and cellular interactions. ${ }^{47-50}$ It is believed that Janus lipid NPs consisting of these nanostructures will provide further benefits such as coencapsulation and release of drugs or face-selective functionalization for cell targeting. However, before the potential of these lipid Janus NPs can be unlocked, a formulation process to produce them consistently and in large quantity is needed. In fact, Tran et al only found less than $10 \%$ of lipid NPs being Janus while the rest of the NPs were monophasic. ${ }^{22}$

\section{Inorganic Janus particles for therapeutics}

Beside polymeric and organic-based Janus NPs, anisotropic particles containing two inorganic compartments have also received increasing level of attention. Among inorganic Janus NPs found in literature, the majority of them were developed based on the inherent therapeutic effects of heavy metals and heavy metal oxides. The Janus functionality not only preserves the original properties of each NP components but also provides new properties not present in single-component NPs due to the interfacial interaction originating from electron transfer across the nanoscale contact. $^{51}$ Moreover, the compartmentalization of Janus NPs allows for the inclusion of fully decoupled modalities, which sometimes have opposite properties, for combined imaging and therapy. For example, well-studied therapeutic strategies involving metallic and oxide NPs include anticancer and antibacterial photothermal therapy (gold NPs AuNPs), ${ }^{52,53}$ gene-therapy siRNA conjugate (AuNPs), ${ }^{54}$ antibacterial chemotherapy (Ag NPs), ${ }^{55,56}$ and magnetic hyperthermia therapy $\left(\mathrm{Fe}_{3} \mathrm{O}_{4} \mathrm{NPs}\right) .{ }^{57,58}$ In addition to these substances, mesoporous silica (MS) with its unique porous structures that can be used to encapsulate drugs has also been considered. ${ }^{59}$ MS NPs are also easily functionalized with antibodies to achieve active targeting of cancer cells. ${ }^{60}$ The main synthetic routes for obtaining inorganic Janus nanoparticles include seed-mediated anisotropic growth, ${ }^{61,62}$ heterogeneous reactions at interfaces, ${ }^{63}$ and deposition and selective annealing of materials with lattice mismatching ${ }^{64}$ Using these strategies, a wide range of metal-metal oxide Janus NPs, including $\mathrm{Au} @ \mathrm{Fe}_{3} \mathrm{O}_{4},{ }^{65,66}$ $\mathrm{Ag} @ \mathrm{Fe}_{3} \mathrm{O}_{4},{ }^{67} \mathrm{Cu} @ \mathrm{Fe}_{3} \mathrm{O}_{4},{ }^{68} \mathrm{Pt} @ \mathrm{Fe}_{3} \mathrm{O}_{4},{ }^{69} \mathrm{Ni} @ \mathrm{Fe}_{3} \mathrm{O}_{4},{ }^{70}$ $\mathrm{Au} @ \mathrm{MnO},{ }^{71}$ and $\mathrm{FePt} @ \mathrm{MnO},{ }^{72}$ has been fabricated and reported. For a more detailed summary on the synthesis of inorganic Janus NPs, the readers are referred to a review article by Schick et al. ${ }^{21}$

In term of using these inorganic Janus NPs for therapeutics, Cao et al created Au@MS Janus NPs which provide dual release of cancer drugs DOX and 6-mercaptopurine (6MP). ${ }^{25}$ The NP synthesis process was based on a modified Pickering emulsion template method $^{73}$ which resulted in the conjugation of 6MP to Au surface and DOX to MS through a gold-thiol interaction and a $\mathrm{pH}-$ responsive hydrazone linker, respectively (Figure 4). With this design, once the NPs enter cancer cells, the linkers will be broken, leading to the release of DOX and 6MP. An in vitro study then demonstrated the enhanced anticancer effect of the dual-loaded Janus NPs against HeLa cells. Interestingly, the authors showed that the release of DOX could be monitored by using Forster resonance energy transfer (FRET) of DOX and 7-hydroxycoumarin-3-carboxylate (CMR). Furthermore, real-time release of $6 \mathrm{MP}$ was observed through the change of surface-enhanced Raman scattering of 6MP. Such dualrelease and real-time monitoring of multiple drugs were believed to be possible due to the special structure of the Janus NPs, which allow for the combination of drastically different chemical or physical properties and directionalities within a single particle.

Chang et al reported the synthesis of Ag@MS NPs that were loaded with cetyltrimethylammonium bromide (CTAB) and tested their antibacterial efficacy with Escherichia coli and Staphylococcus aureus. The results showed that CTAB, a cationic surfactant commonly used as template to synthesize MS NPs, was able to provide synergistic antibacterial activities to $\mathrm{Ag}$ NPs against both types of bacteria. ${ }^{74}$ Following a similar approach, the same group reported Ag@MS NPs for light-activated liver cancer therapy. ${ }^{75}$ The anti-tumor effect was based on the toxicity of $\mathrm{Ag}$ and the photothermal activity of indocyanine green (ICG), which was loaded into the MS compartment (Figure 5A). The NPs were further functionalized with folic acid (FA), which significantly improved cellular uptake in the folate receptor 


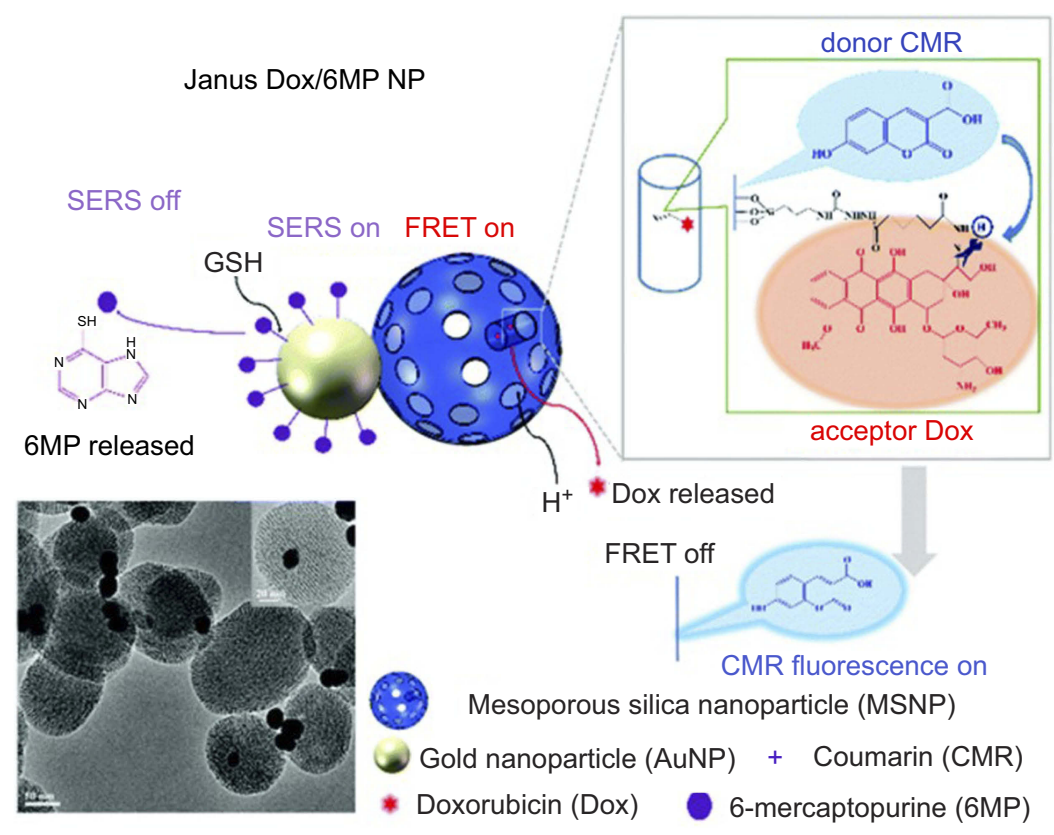

Figure 4 Schematic representation of the Janus Dox/6MP NPs and the mechanism of real-time monitoring of dual drugs with $\mathrm{pH} /$ redox dual responsive release in living cells. Copyright 2016 Royal Society of Chemisty. Reprinted with permission from Cao H, Yang Y, Chen X, Shao Z. Intelligent Janus nanoparticles for intracellular real-time monitoring of dual drug release. Nanoscale. 20I6;8(I2):6754-6760. ${ }^{25}$

(FR)-positive SMMC-7221 human cancer cell line compared to the FR-negative HL-7702 human hepatic embryo cell line. As a result, the Ag-dependent toxicity of the NPs was more pronounced to SMMC-7221 than HL-7702 when the cells were treated with the same dose in vitro. More importantly, a synergistic selective killing effect against SMMC-7221 cells was demonstrated when near-infrared (NIR) light was used to activate ICG. In SMMC-7221 tumor-bearing mice, the synergistic effect of chemo and photothermal therapy using these Janus NPs was again observed with the FAJanus NP@ICG+NIR group displayed the highest tumor inhibition rate at $89 \%$ compared to the PBS control group (Figure 5B and C). This improved anti-tumor efficacy was attributed to the FA active targeting and the simultaneous release of both ICG and silver ions without mutual interference, a feature that was made possible due to the Janus structure.

This synthesis route was proven to be versatile as the same group replaced $\mathrm{Ag}$ with $\mathrm{Fe}_{3} \mathrm{O}_{4}$ and created magnetic Janus NPs. ${ }^{76,77}$ The NPs were loaded with DOX or berberine through $\mathrm{pH}$-sensitive linkers and were used for treating liver cancer. In these cases, the presence of the $\mathrm{Fe}_{3} \mathrm{O}_{4}$ compartment contributes to tumor targeting mediated by the EPR effect and magnetic field-enhanced endocytosis. Ultimately, both in vitro and in vivo studies of subcutaneous and orthotropic liver tumor models in mice showed that the magnetic Janus NPs suppressed of the liver tumor growth and significantly reduced systemic toxicity. It should be noted that prior to these studies core-shell type magnetic MS NPs had been reported. ${ }^{78,79}$ However, several drawbacks of the core-shell structure including considerable interference in magnetic response and limited presence of mesopore formation had led to the development of Janus type magnetic MS NPs. ${ }^{80}$

A notable technique in creating MS-based Janus NPs was reported by $\mathrm{Li}$ et $\mathrm{al},{ }^{81}$ in which they created a single periodic mesoporous organosilica from anisotropic growth on the surface of upconversion NPs (UCNP = NaGdF4:Yb,Tm@ $\left.\mathrm{NaGdF}_{4}\right)$. The synthesis was achieved by first synthesizing a core-shell UCNP-SiO ${ }_{2}$ structure using a reverse microemulsion process (Figure 6A). The second layer of $\mathrm{MS}\left(\mathrm{mSiO}_{2}\right)$ consisted of radial mesoporous channels was formed on the outside of the $\mathrm{CNNP}-\mathrm{SiO}_{2}$ nanoparticles. Finally, a single nanocube crystal of a periodic mesoporous organosilica (PMO) was anisotropically grown on the surface of the outer MS shell of the UCNP using the Stober method. The Janus structure of the NP allows two drugs to be simultaneously loaded and their release independently controlled. In this case, hydrophobic PTX (Guest-1) and hydrophilic DOX (Guest-2) were selected as model drugs to be loaded into the UCNP@SiO $\mathrm{Si}_{2} @ \mathrm{mSiO}_{2} \& \mathrm{PMO}$ Janus NPs (Figure 6B). 


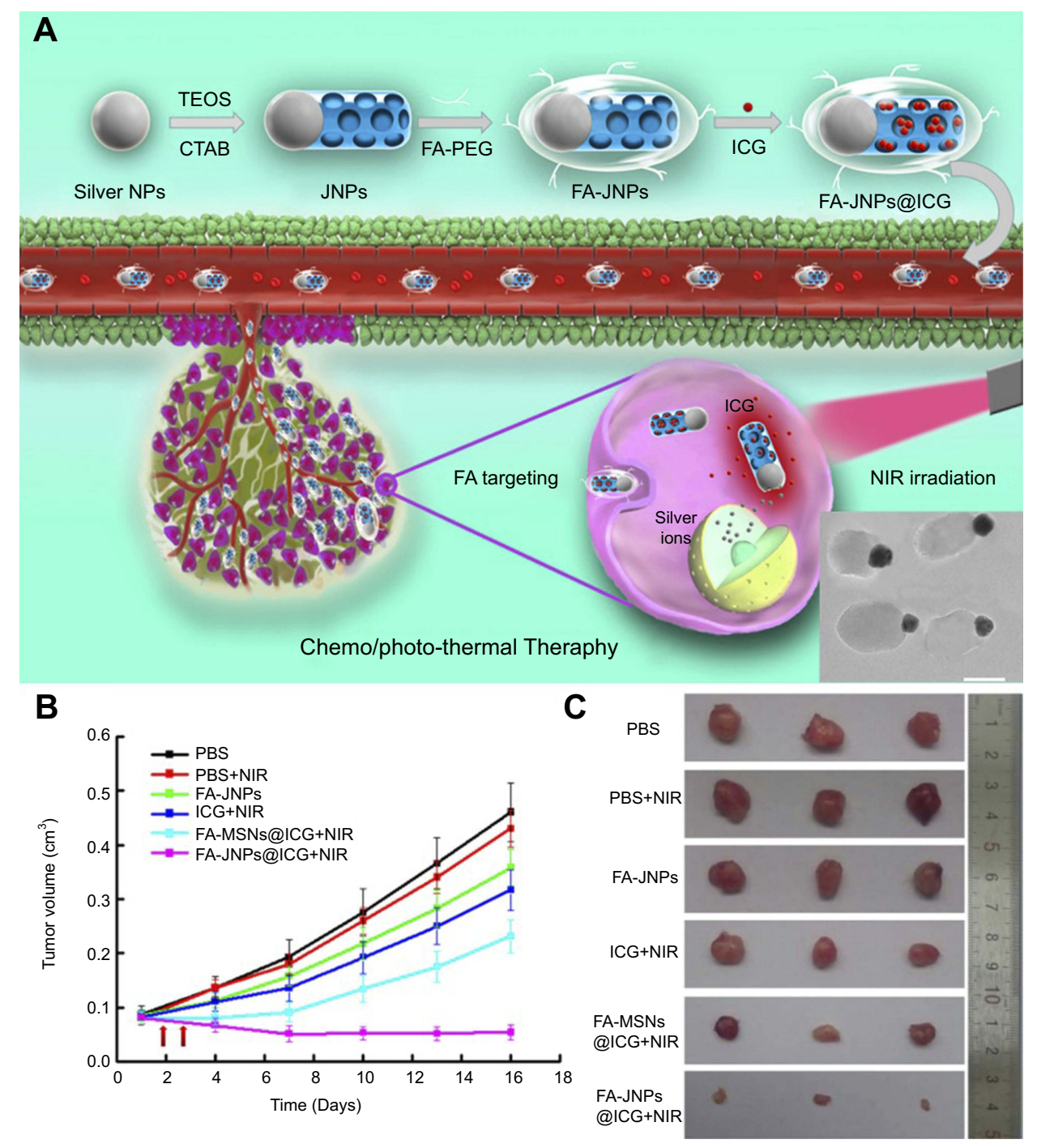

Figure 5 (A) Schematic illustration of the FA-targeting ICG-loaded Ag-MS Janus NPs for synergistic liver cancer chemo/photothermal therapy. Inset: TEM image of the Janus NPs. (B and C) In vivo tumor inhibition evaluation of the chemo/photothermal therapy: (B) SMMC-772I tumor growth curves after various treatments and NIR irradiation at 24- and 48-hr post-injection. (C) Photographs of the excised tumors from mice from each group. Adapted with permission from Wang Z, Chang Z, Lu M, et al. Janus silver/silica nanoplatforms for light-activated liver cancer chemo/photothermal therapy. ACS Appl Mater Interfaces. 20I7;9(36):30306-303 I7. Copyright (20I7) American Chemical Society. ${ }^{75}$

The hydrophilic DOX preferentially adsorbed into the mesoporous channels of the UCNP@ $\mathrm{SiO}_{2} @ \mathrm{mSiO}_{2}$ compartment. Azobenzene, a UV-vis light-sensitive molecule, was used to control the release of DOX from this compartment. On the other hand, PTX absorbed in the mesopore frameworks of the mesoporous PMO crystal compartment. Finally, 1-tetradecanol phase change material was used as another switch to control the release of PTX. This design resulted in Janus NPs with dual-controlled release of two drugs with different solubility. An in vitro study was performed by incubating the nanocomposites with HeLa cells to validate the feasibility of this drug delivery system. The results showed that the Janus NPs with and without drugs did not significantly affect cell viability in the absence of a stimuli source (ie, heat or NIR light). This indicated the negligible amount of drugs released. In contrast, upon a heat treatment $\left(\sim 39^{\circ} \mathrm{C}\right)$ of the Janus NPs, significantly cytotoxicity to the cancer cells ( $>25 \%$ killing efficacy) was observed. This was attributed to the opening of the first switch and the release of the PTX from the PMO compartment. In the presence of both heat and NIR light, PTX and DOX were released at the same time, inducing the further increased cytotoxicity to the cancer cells (over 50\% killing efficacy).

In an attempt to encapsulate and rapidly deliver drug, a self-propelled Janus nanomotor system was developed by Xuan et al. ${ }^{82}$ The $75-\mathrm{nm}$ diameter Janus nanomotor consisted of an MS NP with semi- 


\section{A}
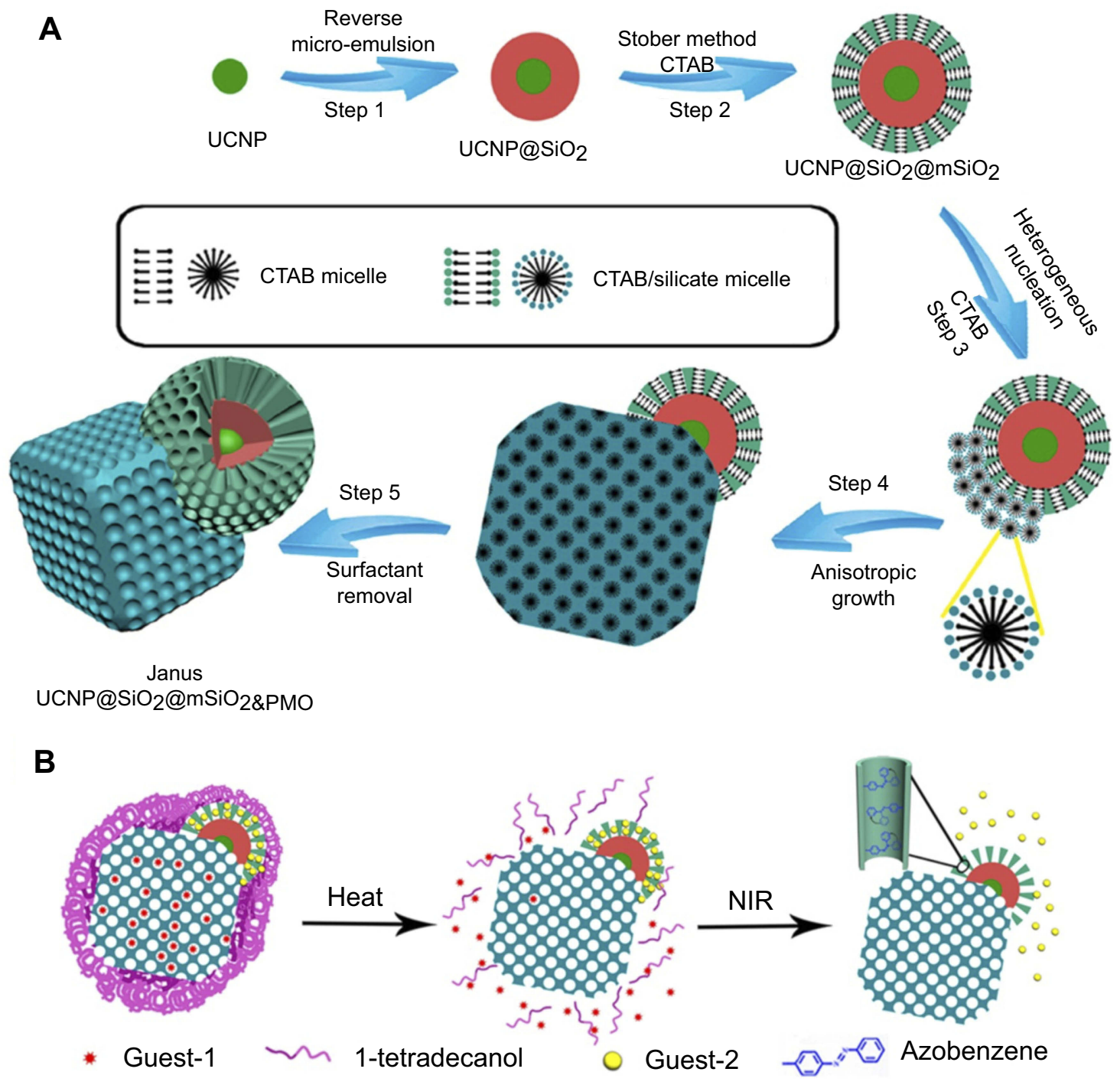

Figure 6 (A) Synthetic procedure for dual compartment Janus mesoporous silica nanocomposites. (B) Schematic illustration for dual-control drug release systems by using the dual-compartment mesoporous Janus nanocomposites. Adapted with permission from Li X, Zhou L, Wei Y, El-Toni AM, Zhang F, Zhao D. Anisotropic growth-induced synthesis of dual-compartment Janus mesoporous silica nanoparticles for bimodal triggered drugs delivery. J Am Chem Soc. 20I4;I36(42):I5086-I5092. Copyright (20I4) American Chemical Society. ${ }^{81}$

hemisphere chromium/platinum metallic caps. In this case, platinum acted as an engine that decomposes hydrogen peroxide to generate oxygen as a driving force to propel the NP. As a proof-of-concept, the NPs were loaded with anticancer drug DOX and coated with phosphatidylcholine (PC) bilayers containing folic acid to enhance the adhesion of the NPs on the surface of cancer cells. The PC bilayers were broken down by phospholipase inside cells, allowing the DOX to be released. One key issue with this system was the toxicity of hydrogen peroxide to mammalian cellular functions, thereby limiting the concentration of hydrogen peroxide in cell culture medium and consequently the maximum speed of the nanomotors. A recent study from the same group showed that by replacing platinum catalysts enzymes, more powerful and efficient polymerbased motors can be achieved, requiring lower peroxide concentrations at physiological temperature. ${ }^{83}$

Inorganic-based Janus NPs have also been explored for non-viral delivery of genes and vaccines. Notable examples are the works involving gold-nickel Janus nanorods from the Leong group. ${ }^{84,85}$ The nanorods, which were fabricated by template synthesis (Figure 7A), were $100 \mathrm{~nm}$ in diameter and $200 \mathrm{~nm}$ in length with $100 \mathrm{~nm}$ gold segments and $100 \mathrm{~nm}$ 


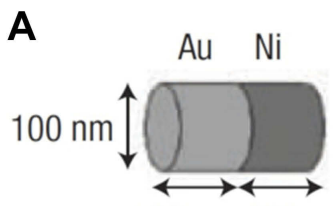

$100 \mathrm{~nm} 100 \mathrm{~nm}$

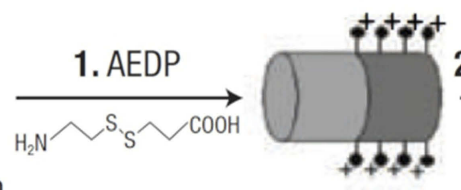

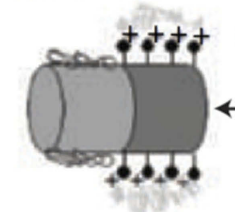

4.
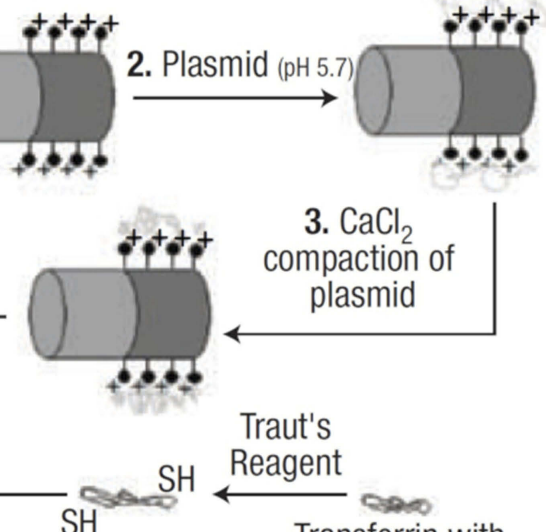

3. $\mathrm{CaCl}_{2}$ compaction of plasmid

Traut's

Reagent

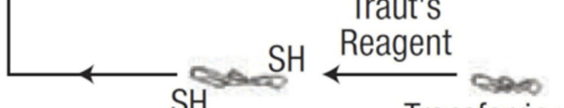

Transferrin with rhodamine tag
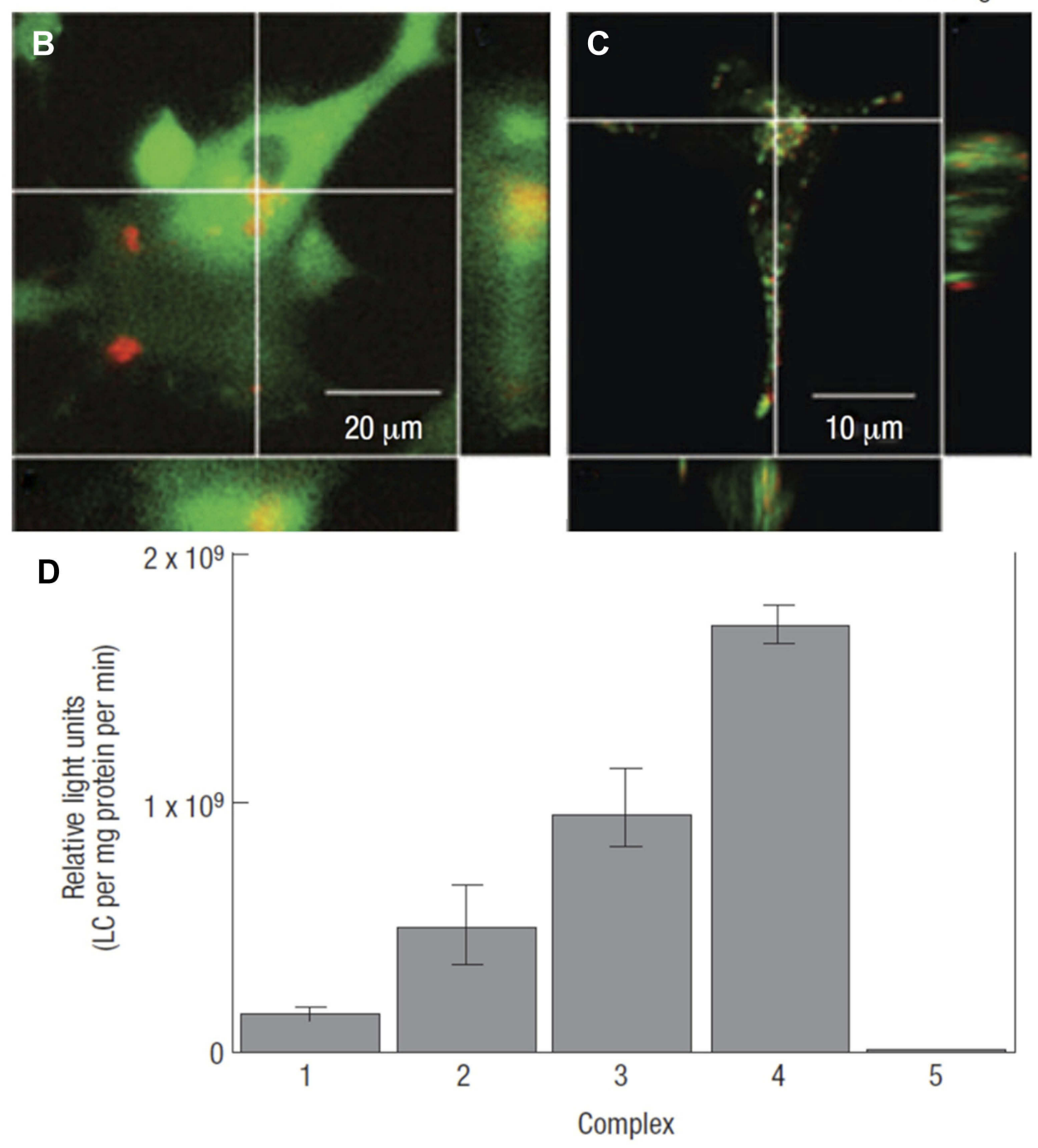

Figure 7 (A) Illustration of Au@Ni Janus nanorod functionalization. (B and C) Confocal microscope images of transfected HEK293 cells. (B) Rhodamine (red-633 nm) identifies the subcellular location of the nanorods whilst GFP expression (green-543 nm) provides confirmation of transfection. Orthogonal sections confirm that the nanorods are within the cell. (C) HEK 293 cell stained with Lysotracker Green shows the location of the nanorods (Rhodamine - red) in relation to acidic organelles in both orthogonal sections. (D) Luciferase (LC) expression of I) nanorod-plasmid complex; 2) nanorod-plasmid/transferrin complex; 3) nanorod-plasmid/transferrin complex incubated with $100 \mu M$ chloroquine; 4) Lipofectamine (positive control); 5) naked DNA (negative control). Adapted with permission from Salem AK, Searson PC, Leong KW. Multifunctional nanorods for gene delivery. Nat Mater. 2003;2 (10):668. ${ }^{85}$ Copyright (C) 2003, Springer Nature. 
nickel segments. The nanorods were modified further by using molecular linkages to attach DNA molecules to the nickel segments and transferrin, a cell-targeting protein, to the gold segments. The results showed that the nanorods were uptaken by the cells and led to much higher transfection efficiency of both GFP and luciferase-encoded plasmids in human embryonic kidney cells (HEK293), especially in the case of transferrin-conjugated nanorods (Figure 7B-D). Preliminary in vivo efficacy studies were performed in the skin and muscle tissues of mice showing vastly different transgene expressions over time. For example, luciferase activity in the skin by nanorods transfection peaked early (830 times higher than background) and decreased quickly to 80 times above background after 4 days. In contrast, intramuscular delivery of the nanorods resulted in only 17 times higher luciferase expression than the background after 1 day, but it was more prolonged, with a luciferase level 85 times above background at day 21. Although promising, further investigation on these nanorods is needed because nickel has toxicity concerns.

\section{Polymer-inorganic composite Janus particles for therapeutics}

Since both polymeric and inorganic materials have their own advantages for therapeutic applications, the combination of polymer and inorganic compartments into a Janus composite NP is logical. ${ }^{86-88}$ The synthesis strategies for creating polymer-inorganic Janus nanoparticles have evolved to provide better precision in controlling anisotropic growth. ${ }^{89}$ In a recent work, Chen et $\mathrm{al}^{90}$ demonstrated the ability to control the anisotropic encapsulation of polymers on metallic NPs by a polymer blocking process, creating so-called regioselective NPs (Figure 8). For this, gold nanospheres (AuNSs - $30 \mathrm{~nm}$ ), amphiphilic diblock copolymer (polystyrene- $b$-polyacrylic acid, PS-PAA) and two ligands in dimethylformamide (DMF)- $\mathrm{H}_{2} \mathrm{O}$ binary solvent were mixed and heated. If only the hydrophobic ligand was used, the PS-PAA blocked the entire surface of the AuNSs, resulting in a fully encapsulated AuNS@PS-PAA core-shell structure. In contrast, when the solution was heated in the presence of the hydrophilic ligand alone, mainly AuNSs without a polymer shell were generated. When both the hydrophobic and the hydrophilic ligand were present in the mixture, the surfaces of AuNSs were partially blocked by the polymer, forming Janus AuNS@PS-PAA particles. Interestingly, the authors showed that the degree of coverage could be tuned by adjusting the solvent ratio and the ligand ratio. The polymer-free areas on the particle surfaces were further modified with single-stranded DNA. Due to the fine selective regional modification, the inter-particle binding potential was directional and selective, resulting in the potential for increasingly complex self-assembly.

An example of this approach is the work performed by Zhang et al in which Au@PAA Janus NPs were first prepared and were then used as templates to grow an MS shell and Au branches separately. The resulting composite Janus NPs went through further PEGylation to improve their stability, conjugation with lactobionic acid (LA) for tumor targeting, and encapsulation with DOX (Figure 9A and B). ${ }^{91}$ Ultimately, the socalled octopus-type Janus NPs (DOX/PEG-OJNP-LA) exhibited $\mathrm{pH}$ and NIR dual-responsive release properties (Figure 9C). The multi-functionalities of the NPs were attributed to their Janus structure, which allowed the NPs more room to perform

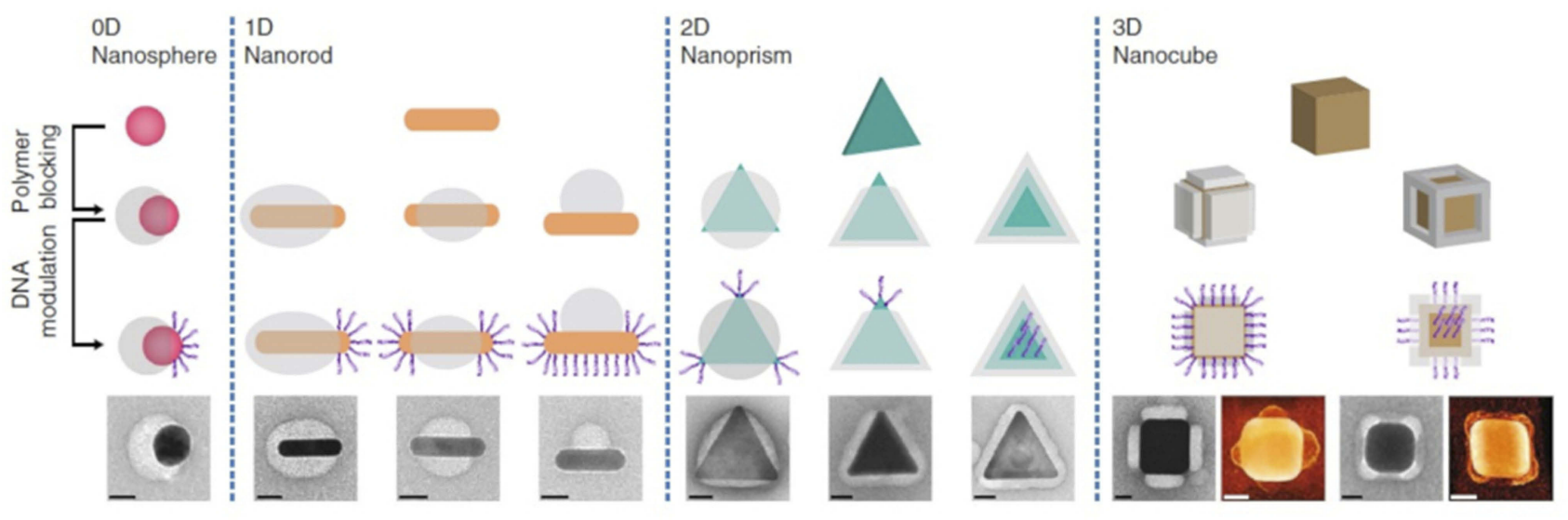

Figure 8 Directional and programmable encapsulated NPs. Top row: colloidal metallic NPs with different sizes, shapes, and compositions, which can be converted into regioselective NPs (rseNPs) through polymer blocking and DNA modification. Second row: controllable polymer blocking will break the homogeneous surface chemistry of NPs, creating specific binding sites on it. Third row: DNA modification on polymer-free surface region provides a specific binding force on the binding site. Bottom row: images of the corresponding rseNPs. Scale bars, $20 \mathrm{~nm}$. Reprinted with permission from Chen G, Gibson KJ, Liu D, et al. Regioselective surface encoding of nanoparticles for programmable self-assembly. Nat Mater. 2018;18:2. Copyright (c) 2018 Springer Nature. ${ }^{90}$ 


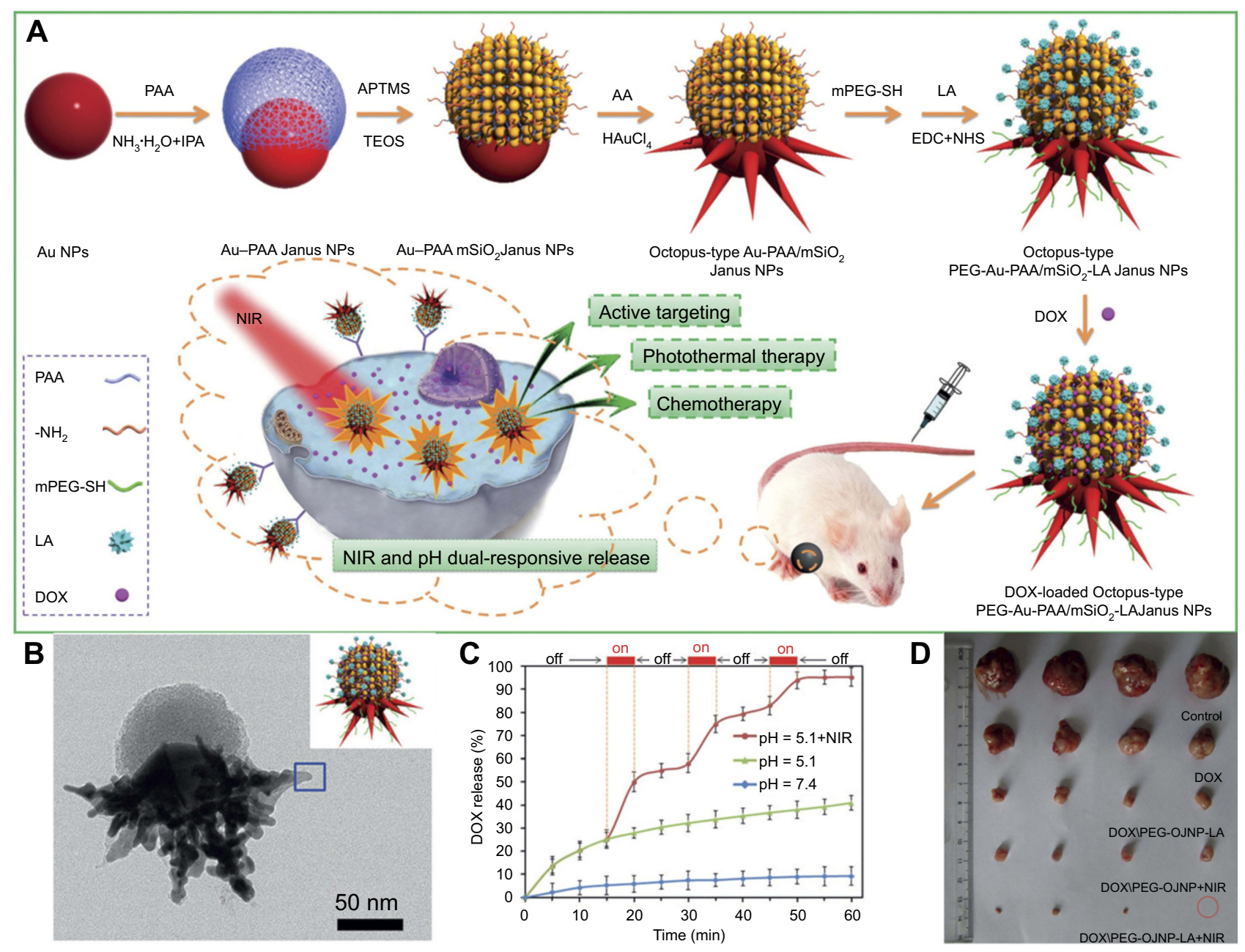

Figure 9 (A) Fabrication of octopus-type PEG-Au@PAA@MS-LA Janus NPs (PEG-OJNP-LA) with pH and NIR light dual-stimuli responsive properties for actively targeted and chemo-photothermal cancer therapy in vitro and in vivo. (B) HRTEM image of a single PEG-OJNP-LA NP. (C) PH and NIR controlled release profiles of DOX/PEGOJNP-LA. (D) Representative photograph of excised H-22 tumors from mice at II days post treatment.Adapted with permission from Zhang L, Chen Y, Li Z, et al. Tailored synthesis of octopus-type janus nanoparticles for synergistic actively-targeted and chemo-photothermal therapy. Angew Chem Int Ed. ${ }^{91}$ Copyright $\odot 2016$ John Wiley and Sons.

different functions for cancer therapy compared with the traditional core-shell NPs. The targeting of LA was proven to be effective by both in vitro cell uptake and in vivo biodistribution experiments in HepG2 cells and H-22 tumor-bearing mice, respectively. Moreover, the NPs when combined with NIR light stimulation showed strong therapeutic effects in vivo in an H-22 tumor mouse model (Figure 9D). The mice treated with DOX/PEG-OJNP-LA upon NIR light showed highest tumor suppression of about $98 \%$, compared to DOX/PEG-OJNP with NIR light (91\%), DOX/PEG-OJNP-LA (81\%), and free DOXtreated group (46\%), implying a synergistic effect of chemo and photothermal therapy. The synthesis concept reported in this work was modified to produce different types of Janus NPs. For example, by replacing Au NPs with palladium nanosheets (PdNS) and MS with metal organic framework (MOF), the same research group were able to produce PdNS/MOF Janus NPs, which were further engineered with cyclodextrin to encapsulate both hydrophobic 10-hydroxycampothecin and hydrophilic DOX. The dual drug-loaded Janus NPs demonstrated synergistic hydrophobic and hydrophilic chemotherapy and photothermal therapy in vitro and in vivo. ${ }^{88}$

Recently, Shaghaghi et al ${ }^{92}$ reported superparamagnetic iron oxide nanoparticle (SPION)-based multifunctional Janus composite NPs designed to carry DOX through the blood-brain barrier (BBB) to treat brain cancer glioblastoma multiforme. The synthesis of these Janus NPs appeared to be fairly complicated with 4 main steps: 1) preparation of the core particle, 2) the hydrophilic part, 3) the hydrophobic part, and 4) conjugating both sides on a NP (Figure 10). 1) In the first step, bare SPIONs were masked and immobilized in 

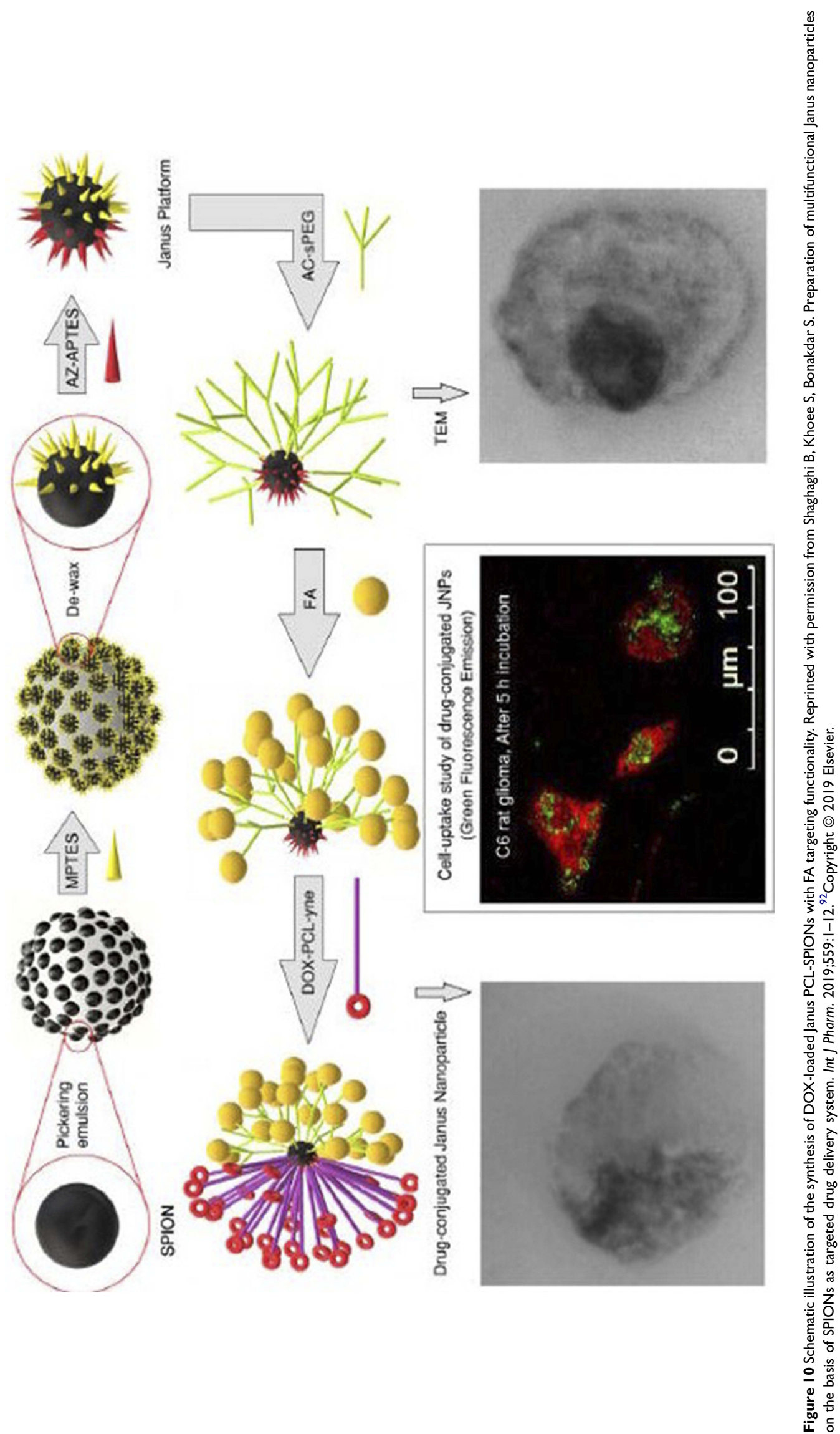
paraffin wax particles, where the unshielded side of waxed SPIONs was grafted with (3-mercaptopropyl) triethoxysilane (MPTES), creating the hydrophilic side of the NPs. After dewaxing, the bare side of the SPION-SH was grafted with azidated (3-aminopropyl) triethoxysilane (AZ-APTES) creating the hydrophobic side. 2) In step two, the hydrophilic side of the SPION was prepared by synthesizing mono(acryloyl) functionalized star-shaped PEG (Acl-sPEG), where FA would later be conjugated onto. 3) The hydrophobic side was prepared by conjugating DOX to poly( $\varepsilon$ caprolactone) (PCL), which was modified with a free alkyne group. 4) Acl-sPEG was conjugated to the core particles via thiol-ene click reaction. FA was then conjugated to the AclsPEG part by carbodiimide coupling. Finally, the hydrophobic side DOX-PCL-yne was conjugated to the core particles via alkyne-azide cycloaddition click reaction. The authors showed that the release of DOX was $\mathrm{pH}$ dependent with the cumulative release of DOX at $\mathrm{pH}=6.2$ was $25 \%, 32 \%$, and $42 \%$ after 24,50 , and $256 \mathrm{hrs}$, respectively, compared to $1 \%$, $2.3 \%$, and $9.5 \%$ in the case of $\mathrm{pH}=7.4$. This led to somewhat selective in vitro cytotoxicity against rat C6 glioma cells caused by the Janus NPs at $\mathrm{pH}=6.2$ compared to $\mathrm{pH}=7.4$. Cell uptake study showed that after $5 \mathrm{hrs}$ of incubation with folate receptor expressed C6 glioma cells, the Janus NPs were internalized at a much higher level compared to the FAfree Janus NPs, indicating the effectiveness of the FA targeting functionality. Unfortunately, the study did not examine the possibility for these NPs to cross the BBB.

\section{Janus particles for in vivo imaging}

Medical imaging plays important roles in disease detection, prognosis, and treatment planning. Major medical imaging techniques include optical imaging, X-ray computed tomography (CT), magnetic resonance imaging (MRI), positron emission tomography (PET), singlephoton emission CT (SPECT), and photo-acoustic (PA) imaging. For many of these techniques, a contrast agent is needed to improve the image quality. Additionally, due to each imaging method has its own benefits and limitations, there have been a strong interest in combining complement imaging modalities into a single platform. The intrinsic physicochemical properties of inorganic NPs make them suitable contrast agents for optical imaging, MRI, CT, and PA imaging. By incorporating small molecules, these NPs can find applications in nearly all imaging modalities. Due to their anisotropic structure, Janus NPs have the advantage of comprising two different faces, which not only enable two complementary imaging modalities but also offer two functional surfaces for the attachment of different kinds of molecules, making them especially attractive as multifunctional probes.

A few studies have utilized the CT and PA imaging capabilities of gold and MRI contrast enhancement of iron oxide to create multi-modal imaging Janus NPs. ${ }^{20,65,93,94}$ For example, Reguera et al reported Janus gold-iron oxide NPs as contrast agents for multimodal imaging. ${ }^{93}$ The synthesis of these Janus NPs started with the formation of AuNSs that were used as seeds for $\mathrm{Au} @ \mathrm{Fe}_{3} \mathrm{O}_{4}$ nanodumbbells, which were subsequently used as seeds for the directional growth of asymmetric Au nanostars. Figure 11 shows representative TEM images corresponding to NPs formed at different nanodumbbell seed concentrations (and constant amount of gold precursor). Varying this parameter resulted in NPs with average diameters ranging from $\sim 20$ $\mathrm{nm}$ to $\sim 50 \mathrm{~nm}$. The study demonstrated the use of these Janus NPs in several imaging techniques, such as $T_{2^{-}}$ weighted MRI, X-ray CT, PA imaging, optical imaging under bright and dark field illumination, transmission electron microscopy (TEM), and surface-enhanced Raman spectroscopy (SERS) imaging. Compared with core-shell NPs, the Janus morphology resulted highly beneficial in several aspects, offering high availability of the iron oxide surface, which consequently gives rise to high $r_{2}$ relaxivity values and Prussian blue staining ability. On the other hand, when compared with conventional Janus dumbbelllike NPs, the nanostar morphology enables their use in PA and SERS imaging in the optical NIR biological window.

Dumbbell-shaped inorganic Janus NPs have also attracted much attention as multifunctional probes for diagnostic and therapeutic applications. For example, $\mathrm{Xu}$ et al reported dumbbell gold-iron oxide NPs, which were coated with a layer of oleate and oleylamine. ${ }^{65}$ The dumbbell structure was formed through epitaxial growth of $\mathrm{Fe}_{3} \mathrm{O}_{4}$ on the Au seeds, a process that allowed fine control over the size of both $\mathrm{Fe}_{3} \mathrm{O}_{4}$ and $\mathrm{Au}$ particles. To demonstrate the ability to modify of the two surfaces of the Janus NPs independently, the authors functionalized epidermal growth factor receptor antibody (EGFRA) linked PEG-dopamine to the $\mathrm{Fe}_{3} \mathrm{O}_{4}$ side and HS-PEG-NH $\mathrm{N}_{2}$ to the $\mathrm{Au}$ side through ligand exchange reactions (Figure 12). The presence of EGFRA also aided the binding of the Janus NPs to EGFR overexpressing cancer cells such as A431 human epithelial carcinoma cells. The dumbbell NPs were demonstrated to be capable of imaging the exact same tissue area through both MRI and an optical source without the fast signal loss observed in the common fluorescent labeling. It was 


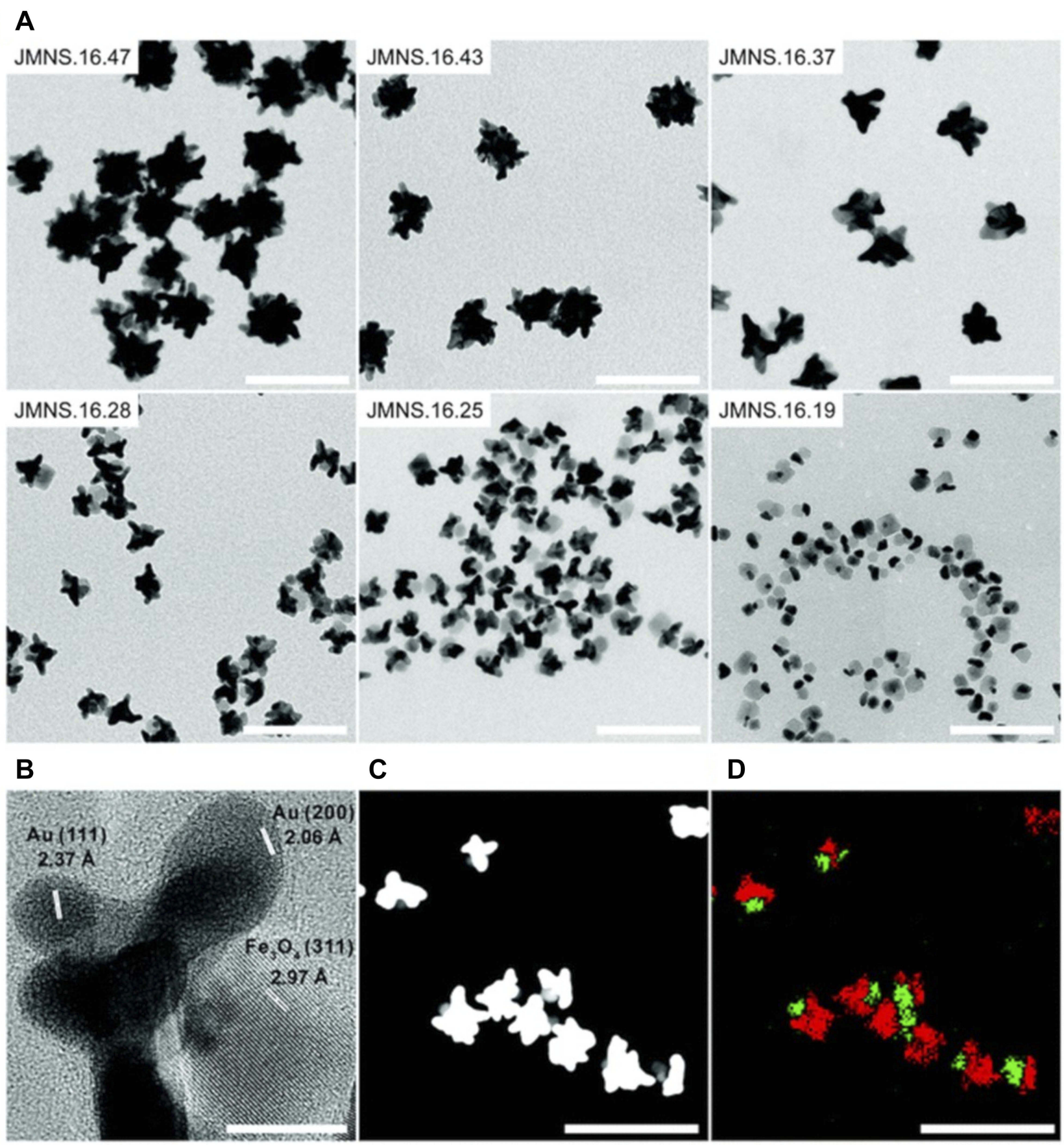

Figure II (A) TEM images of different Janus magnetic nanostars (JMNSs) obtained by varying the ratio of nanodumbbells-seeds to gold salt. As the NP size decreases, the iron oxide part (light grey) can be distinguished from the gold domain (dark grey or black). (B) High-resolution TEM image of an NP (JMNS. I6.28), whose average diameter of the iron oxide part was $16 \mathrm{~nm}$ and that of the whole NP was $28 \mathrm{~nm}$, showing single crystal nanostar tips and Fe $\mathrm{O}_{4}$. (C) HAADF-STEM image of (JMNS. I6.28), where gold appears white while iron oxide appears gray. (D) EDX mapping of Fe (green) and Au (red) domains in (C). (Scale bars for (A), (C) and (D)=100 nm; scale bar for (B) $=10$ $\mathrm{nm}$ ). Reprinted with permission from Reguera J, de Aberasturi DJ, Henriksen-Lacey M, et al. Janus plasmonic-magnetic gold-iron oxide nanoparticles as contrast agents for multimodal imaging. Nanoscale. 2017;9(27):9467-9480. ${ }^{93}$ Copyright 2017 Royal Society of Chemistry.

suggested that they could be used to achieve high sensitivity in diagnostic imaging applications.

Gold and iron oxide dumbbell Janus NPs were also explored as dual-modality imaging probes for both $\mathrm{CT}$ and MRI. ${ }^{20} \mathrm{~A}$ thermal decomposition process was utilized to synthesize $\mathrm{Au}-\mathrm{Fe}_{3} \mathrm{O}_{4}$ dumbbell particles from mixtures of Fe-oleate and Au-oleylamine complexes. The NPs were further coated with an amphiphilic polymer, poly(DMA-r-mPEGMA-r-MA), to improve their stability in physiological conditions and reduce nonspecific 

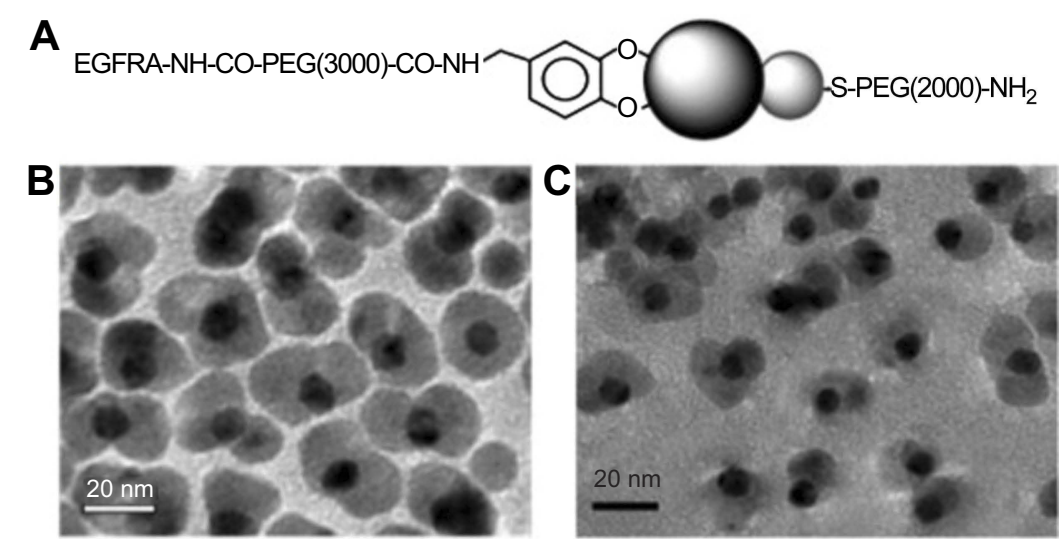

Figure 12 (A) Schematic illustration of surface functionalization of the $\mathrm{Au}_{-} \mathrm{Fe}_{3} \mathrm{O}_{4} \mathrm{NPs}$. (B and $\left.\mathbf{C}\right) \mathrm{TEM}$ images of the 8-20 nm Au-Fe $\mathrm{O}_{4}$ particles before (B) and after $(\mathbf{C})$ surface modification. Reprinted with permission from Xu C, Xie J, Ho D, et al. Au- $\mathrm{Fe}_{3} \mathrm{O}_{4}$ dumbbell nanoparticles as dual-functional probes. Angew Chem Int Ed. 2008;47 (I): 173-176..$^{65}$ Copyright (C) 2008 John Wiley and Sons.

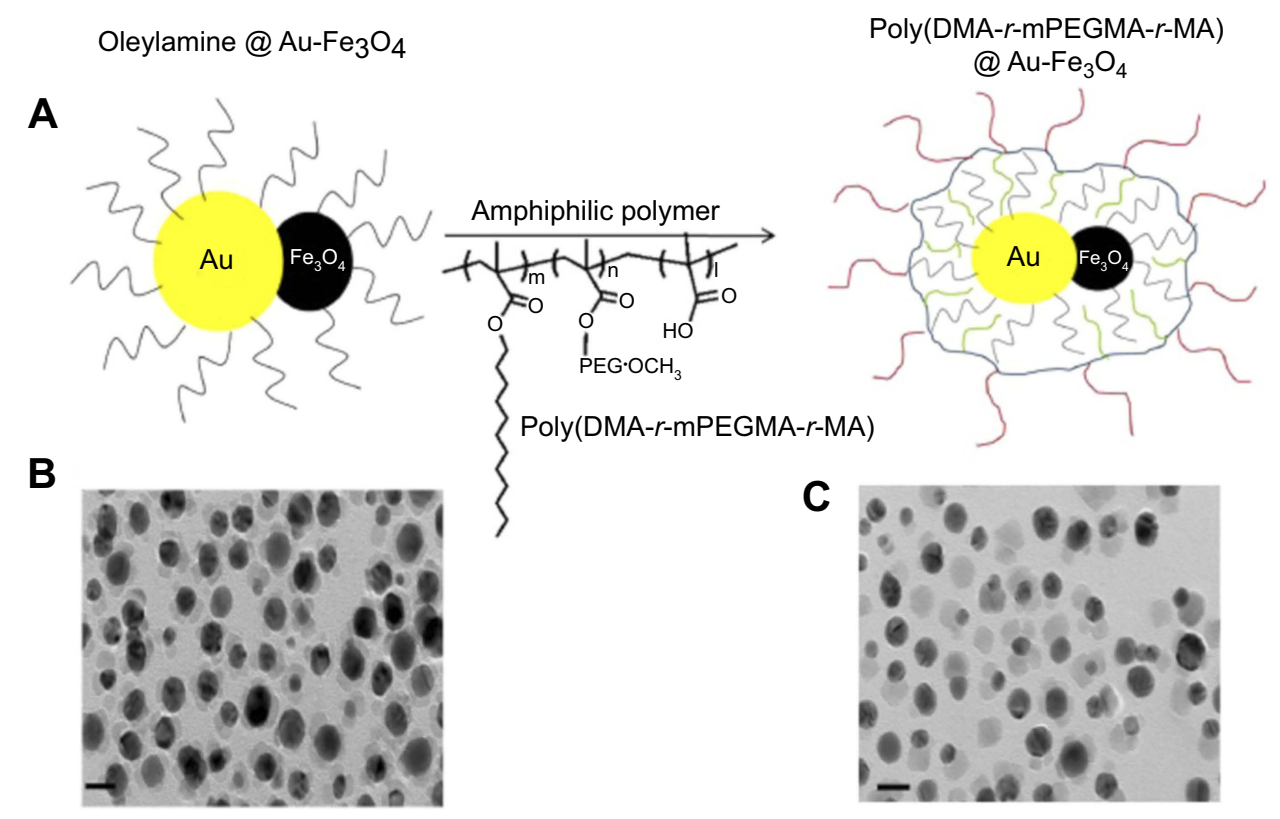

Figure 13 (A) Schematic illustration of the poly(DMA-r-mPEGMA-r-MA) coating of the hybrid gold-iron oxide (Au-Fe $\left.\mathrm{O}_{4}\right) \mathrm{NPs}_{\text {. }}(\mathbf{B}),(\mathbf{C})$ TEM images of the hybrid Au-Fe 3 $\mathrm{O}_{4} \mathrm{NPs}$ before (B) and after (C) coating with poly(DMA-r-mPEGMA-r-MA). The scale bar in the TEM image indicates $20 \mathrm{~nm}$. Reprinted with permission from Kim D, Yu MK, Lee TS, Park JJ, Jeong YY, Jon S. Amphiphilic polymer-coated hybrid nanoparticles as CT/MRI dual contrast agents. Nanotechnology. 20II;22(I5):I55I0I. ${ }^{20}$ Copyright 20II IOP Publishing.

adsorption of proteins (Figure 13). The study showed that the hybrid NPs had high CT attenuation, due to the constituent gold NPs, and provided a good MR signal, as a result of the contained iron oxide NPs. Interestingly, the Janus NPs outperformed pure Au NPs in CT and commercial iron oxide-based contrast agent Resovist in MRI testing, respectively. This was attributed to the synergistic effect seen when both $\mathrm{Au}$ and $\mathrm{Fe}_{3} \mathrm{O}_{4}$ were present within a single particle. More importantly, the NPs demonstrated dual contrast capability in vivo when intravenously injected into hepatoma-bearing mice. The animal micro-CT and $3 \mathrm{~T}$ MRI results showed high contrast between the hepatoma and normal hepatic parenchyma in both CT and MRI.

Superparamagnetic manganese oxide $(\mathrm{MnO})$ has also been considered to combine with Au to form Janus NPs. In a study by Schick et al, the Au-MnO Janus NPs were prepared by a seed-mediated nucleation and growth method, which allowed precise control over domain sizes, surface functionalization, and dye labeling (Figure 14A and B). ${ }^{71}$ $\mathrm{Au}$ NPs possess properties that make them promising as optical imaging probes, contrast agents for CT, and twophoton photoluminescence probes. MnO NPs were selected because unlike $\mathrm{Fe}_{3} \mathrm{O}_{4}$, which are mostly used as $T_{2}$ contrast 


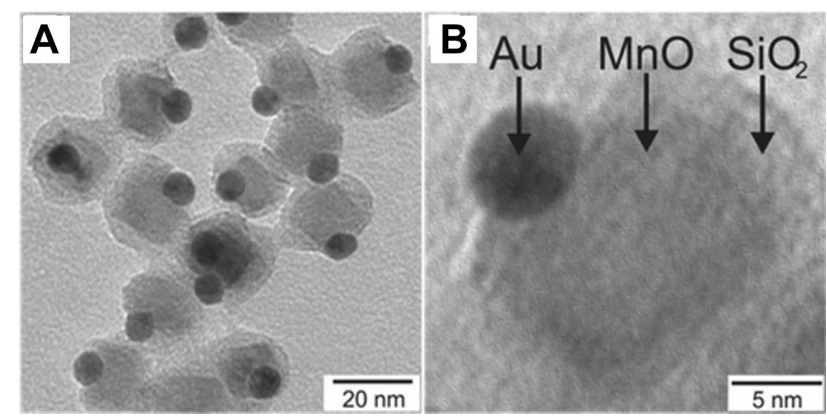

Figure 14 (A) Overview TEM bright field image of Au@MnO@SiO ${ }_{2}$ NPs, (B) TEM micrograph of a single Au@MnO@SiO 2 particle. Reprinted with permission from Schick I, Lorenz S, Gehrig D, et al. Multifunctional two-photon active silica-coated Au@ MnO Janus particles for selective dual functionalization and imaging. J Am Chem Soc. 2014;136(6):2473-2483. ${ }^{71}$ Copyright 2014 American Chemical Society.

probes for MRI, MnO NPs induce hyperintensities on $T_{1^{-}}$ weighted MRI maps. A silica coating was applied on the $\mathrm{MnO}$ domain to improve the particle's biocompatibility and aqueous stability, and to provide a platform for further biomolecule conjugation (eg, antibodies, proteins). Timeresolved fluorescence spectroscopy in combination with confocal laser scanning microscopy (CLSM) revealed the Janus NPs to be highly two-photon active due to the presence of the $\mathrm{Au}$ domain. The in vitro biocompatibility assay results showed that the Janus particles were non-cytotoxic against human renal carcinoma cells (Caki) and HeLa cells at up to $100 \mu \mathrm{g} / \mathrm{mL}$. Additionally, confocal fluorescent and twophoton imaging were demonstrated using the Au-MnO Janus NPs and HeLa cells in vitro. However, the study did not explicitly show the magnetic property and MRI contrast capability of the synthesized Janus NPs.

\section{Janus NPs for theranostics}

Theranostic NPs are developed to combine their capability in therapeutics and diagnostics/imaging to maximize the efficacy of a therapy. So far, most Janus NPs for theranostics have been either inorganic-inorganic or polymer-inorganic composite. Examples of inorganic-based Janus NPs are the Ag@MS and Au@MS NPs developed by Shao et al and Wang et al. ${ }^{95,96}$ These NPs were synthesized using a similar approach that was reported previously by the same group which used tetraethyl orthosilicate (TEOS) as silica source, CTAB as a template, and either Ag NPs or Au nanorods as the substrate, respectively, ${ }^{97}$ The Ag-MS NPs were loaded with DOX through a $\mathrm{pH}$-sensitive linker for selective chemotherapy, while SERS activity was used for imaging and monitoring drug release. ${ }^{95}$ On the other hand, the Au@MS NPs utilized the photothermal activity of gold for cancer therapy and the addition of fluorescent compound Rhodamine B to the MS matrix for cellular imaging. ${ }^{96}$ Both types of NPs were tested in vitro against HepG2 liver cancer cells and were routinely found to enter cells to realize cellular imaging and demonstrate significant chemotoxicity and photothermal effects, respectively. In addition to HepG2 cells, the cytotoxicity of Ag@MS Janus NPs was further tested with other cancer cell lines including lung cancer A549 and breast cancer MCF-7 and normal cell lines including hepatic embryo cell line HL-7702, human umbilical vein endothelial cells (HUVEC) and bone marrow mesenchymal stem cells (BMSCs). Although dose-dependent toxicity profiles were observed in all tested cell lines, the DOX-loaded Ag-MS Janus NPs displayed significantly lower damage to normal cells compared to cancer cells. The selective toxicity of these NPs was attributed to the $\mathrm{pH}$-sensitively drug release behavior, as well as the higher endocytosis capacity of the NPs in tumor cells.

Another strategy to achieve theranostics is by combining the property of magnetic NPs as a contrast agent for MRI with photothermal or photodynamic therapy. Additionally, a chemotherapeutic agent maybe incorporated to further improve the efficacy of the system. Commonly used MRI capable NPs include iron oxide $\left(\mathrm{Fe}_{3} \mathrm{O}_{4}\right),{ }^{98,99}$ iron carbide $\left(\mathrm{Fe}_{3} \mathrm{C}\right),{ }^{27} \mathrm{Fe}(\mathrm{OH})_{3},{ }^{100} \mathrm{MnO}_{2},{ }^{101}$ and $\mathrm{Mn}_{3} \mathrm{O}_{4} \cdot{ }^{102}$ For example, a recent work by $\mathrm{Li}$ et al reported multifunctional $\mathrm{CuS} @ \mathrm{Au} @ \mathrm{MnO}_{2}$ ternary Janus NPs, which consisted of a hemispherical $\mathrm{MnO}_{2}$ at one side and an Au core covered with a $\mathrm{CuS}$ shell at the opposite side. ${ }^{101}$ Besides the CT imaging capacity of Au NPs, the NPs exhibited photothermal conversion and $T_{1}$-weighted MRI capabilities in the $\mathrm{CuS}$ and $\mathrm{MnO}_{2}$ domains, respectively. Furthermore, the NPs were coated with PEG to improve stability and a hydrophobic model drug, Celastrol, was encapsulated in the $\mathrm{MnO}_{2}$ porous compartment and demonstrated both $\mathrm{pH}$ and NIR irradiation-dependent release profiles (Figure 15A). The authors demonstrated a synergistic anticancer therapy in vitro and in vivo in HepG2 cells and H-22 hepatocarcinoma tumor bearing mice, respectively (Figure 15B-F). 

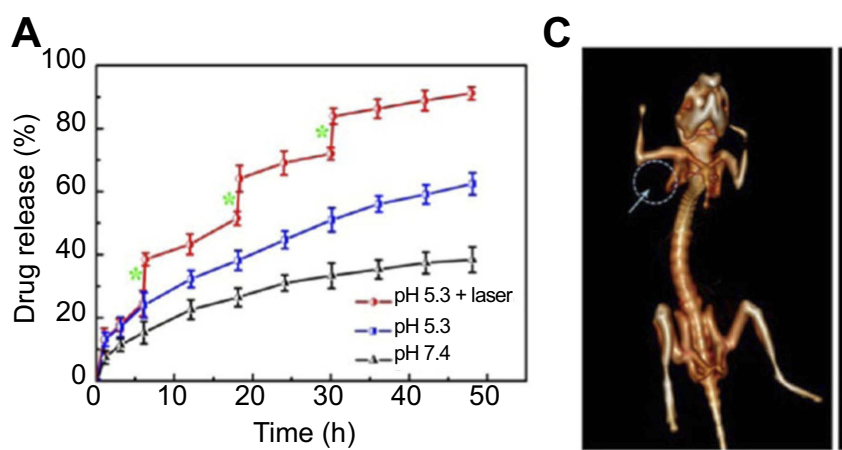

Before injection

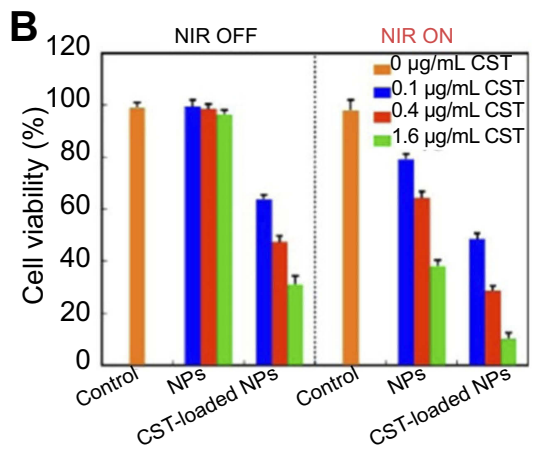

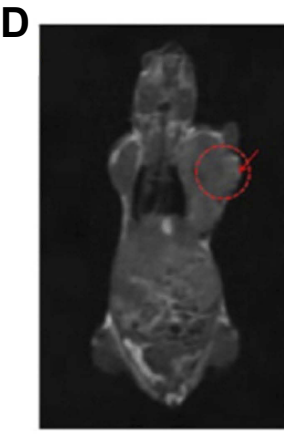

Before injection

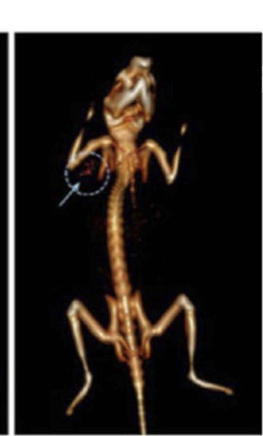

After injection

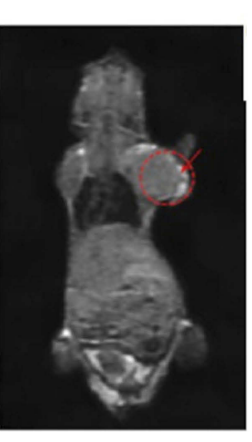

After injection
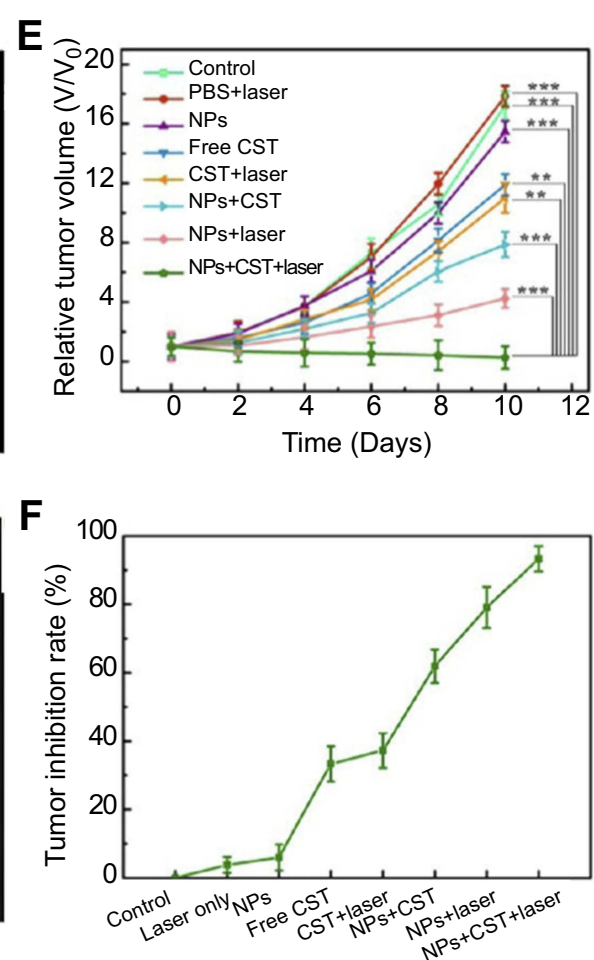

Figure 15 (A) Release profiles of Celastrol (CST)-loaded PEG-CuS@Au@MnO ternary Janus NPs in PBS at pH 7.4 and pH 5.3 with and without laser irradiation (time periods under irradiation are labeled by green stars). (B) Cytotoxicity of HepG2 cells after incubation with various concentrations of Janus NPs treated with or without NIR irradiation. (C and $\mathbf{D})$ In vivo CT and MR images of mice before and after injection with PEG-CuS@Au@MnO 2 Janus NPs for 24 hrs. (E, F) Tumor growth curves and tumor inhibition rate of $\mathrm{H}-22$ tumor-bearing mice subjected to various types of Janus NPs and treatment conditions $* * p<0.0 \mathrm{I}$, *** $p<0.00 \mathrm{I}$. Adapted with permission from Li $\mathrm{S}$, Zhang L,ChenX, et al. Selective growth synthesis of ternary Janus nanoparticles for imaging-guided synergistic chemo- and photothermal therapy in the second NIRwindow. ACS Appl Mater Interfaces. 2018;101470 (28):24137-24148. ${ }^{101}$ Copyright 2018 American Chemical Society.

Several polymeric-inorganic composite Janus NPs for theranostics have been reported by the $\mathrm{Li}$ and Wang groups. ${ }^{100,103,104}$ Notably, they demonstrated a synthetic strategy for the fabrication of polydopamine/ mesoporous calcium phosphate (PDA@mCaP) hollow Janus NPs, which were further decorated with PEG for stability and ICG for photothermal activity on the PDA domain (Figure 16). ${ }^{104}$ The mCaP compartment with hollow cavities was utilized as storage and release DOX. The resulted NPs, PEG-ICG-PDA@mCaP H-Janus NPs, exhibited a combined activity of chemo and photothermal therapy, as well as NIR and $\mathrm{pH}$ dual-responsive drug release property and PA imaging capability. In fact, after 5 mins of irradiation with a NIR laser $\left(1 \mathrm{~W} \mathrm{~cm}^{-2}\right)$, the Janus NP solution could be heated up to $56.5^{\circ} \mathrm{C}$ demonstrating high photothermal activity while the pure water only increased by $1.8^{\circ} \mathrm{C}$ (Figure 16F). Additionally, the Janus NPs displayed high IR thermal imaging property, which made them suitable for monitoring the NPs treatment in vivo (Figure 16G). More importantly, the combination of DOX-loaded Janus NPs with NIR irradiation exhibited significantly better anti-tumor activity in HepG-2 tumor-bearing mice compared to other monotherapies without DOX or without laser. The combined treatment resulted in an impressive $96.2 \%$ tumor suppression while not causing any damage to other major tissues.

In an example of inorganic-based Janus NPs, Ju et al synthesized $12 \mathrm{~nm}$ monodisperse $\mathrm{Au} @ \mathrm{Fe}_{2} \mathrm{C}$ anisotropic NPs for imaging-guided cancer therapy (Figure 17). ${ }^{27}$ The study utilized the advantage of Au NPs as a photothermal therapy (PTT) agent. Additionally, $\mathrm{Au}$ and $\mathrm{Fe}_{2} \mathrm{C}$ component were used as triple-modal $\mathrm{MRI} / \mathrm{CT} /$ multispectral photoacoustic tomography (MSOT) imaging agents. The Janus structure of the NPs allowed them to express multiple functions simultaneously and interpedently. In vitro, the Janus NPs showed a significant photothermal effect with a $30.2 \%$ calculated photothermal transduction efficiency under $808 \mathrm{~nm}$ laser irradiation. The $\mathrm{Au} @ \mathrm{Fe}_{2} \mathrm{C}$ Janus NPs were further conjugated with affibody proteins $Z_{\mathrm{HER} 2: 342}$ to improve uptake by cells that overexpressed HER2 receptors such as human mammary carcinoma MDA-MB-231 cells. When the NPs were exposed to laser stimulation, much lower MDA-MB-231 cell viability was observed, especially in the case of $\mathrm{Au} @ \mathrm{Fe}_{2} \mathrm{C}-\mathrm{Z}_{\mathrm{HER} 2: 342}$, compared to cells treated with only NPs or only laser. Moreover, the authors found that $\mathrm{Au} @ \mathrm{Fe}_{2} \mathrm{C}-\mathrm{Z}_{\mathrm{HER} 2: 342} \mathrm{NPs}$ have more accumulation and deeper penetration in tumor sites 

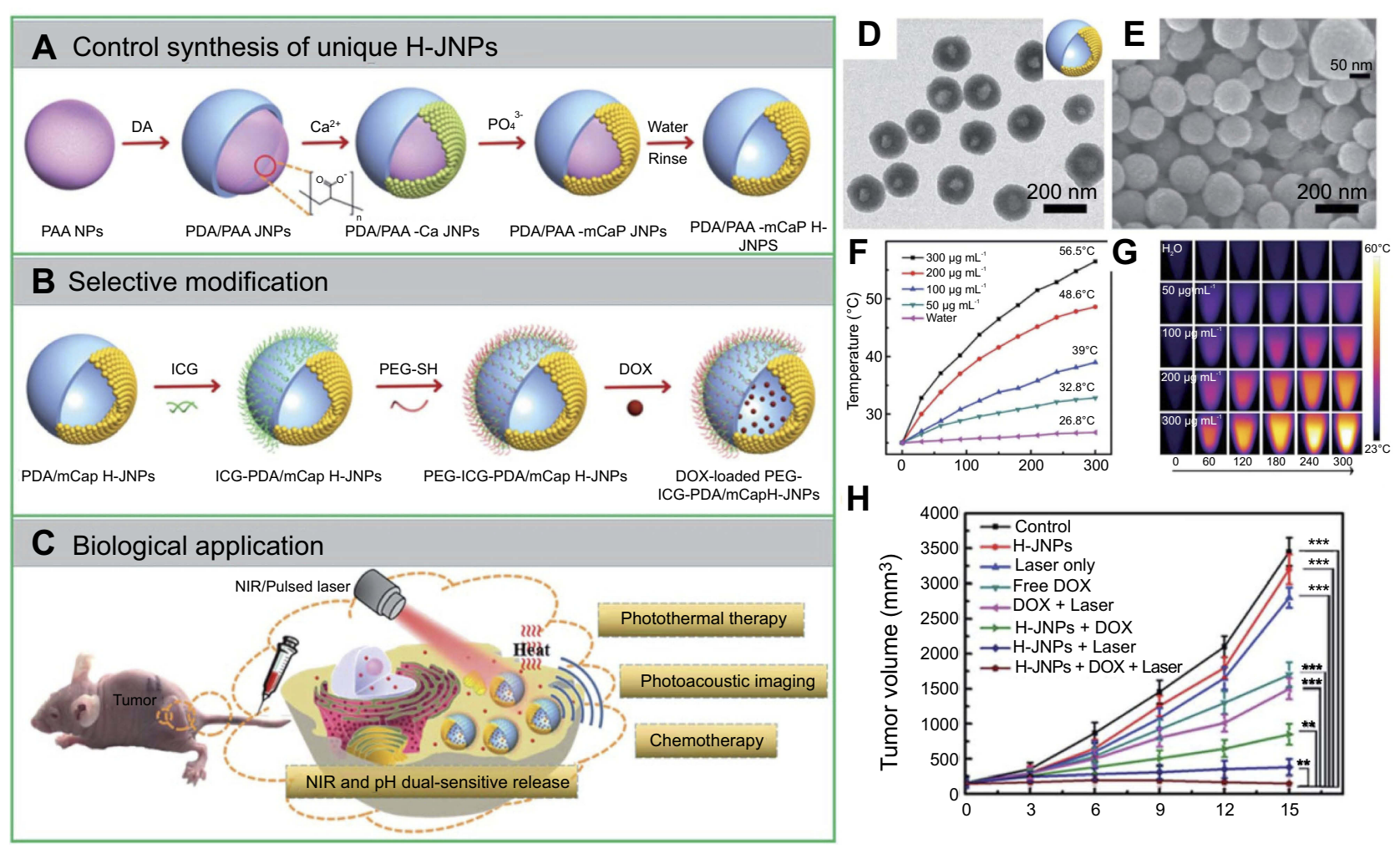

Figure 16 A schematic showing (A, B) the controlled synthesis and functionalization of the PEG-ICG-PDA@mCaP hollow Janus NPs and (C) the PA imaging-guided chemophotothermal synergistic cancer therapy. (D, E) TEM and SEM of the PDA/mCaP hollow Janus NPs. (F, G) Temperature elevation of the hollow Janus NPs when irradiated with NIR laser $\left(\mathrm{I} \mathrm{W} \mathrm{cm}{ }^{-2}\right)$ and the corresponding infrared thermal images. $(\mathbf{H})$ Tumor growth curve of HepG-2 bearing mice treated with DOX-loaded hollow Janus NP and NIR laser. ** $<<0.0 \mathrm{I}$, $* * *$ p $<0.00 \mathrm{I}$. Adapted with permission from Zhang M, Zhang L, Chen Y, Li L, Su Z, Wang C. Precise synthesis of unique polydopamine/mesoporous calcium phosphate hollow Janus nanoparticles for imaging-guided chemo-photothermal synergistic therapy. Chem Sci. 2017;8(I2):8067-8077. ${ }^{104}$ Copyright 2017 Royal Society of Chemistry.

than non-targeting Janus NPs $\left(\mathrm{Au} @ \mathrm{Fe}_{2} \mathrm{C}-\mathrm{PEG}\right)$ in vivo. PTT results in vivo using MDA-MB-231 tumor-bearing mice, which were injected with $\mathrm{Au} @ \mathrm{Fe}_{2} \mathrm{C}-\mathrm{Z}_{\mathrm{HER} 2: 342} \mathrm{NPs}$, demonstrated that the NPs were highly effective in inhibiting and eliminating tumor while causing negligible abnormality in other major organs. The distribution of Janus NPs in tumors and other organs were obtained from a combination of $T_{2^{-}}$ weighted MRI, MSOT, and CT. Additionally, the heating of the NPs and tumor locally was monitored by thermal IR imaging. Collectively, it is clear that the $\mathrm{Au} @ \mathrm{Fe}_{2} \mathrm{C}-\mathrm{Z}_{\mathrm{HER} 2: 342}$ Janus NPs had great potential as precision theranostic nanomedicines.

\section{Janus particles for biosensing applications}

In addition to applications in therapeutics and in vivo imaging, Janus particles have also been developed as biosensors. The existing literature can be classified into two categories. One category involves utilizing the compartmentalization within Janus particles into different domains, which serve as independent biological recognition and signal transduction modalities. The other category relies on the "on-the-fly" detection of analytes by selfpropelled Janus particles, which can significantly improve the response time of the sensors. Compared to the second category of moving biosensors, the first type of Janus particles can be regarded as static.

\section{Static Janus particles for biosensing}

Isotropic NPs have been studied widely as biosensors. The surfaces of these NPs are often functionalized with large biomolecules such as proteins or DNAs to provide binding sites for analytes. However, the presence of these biomolecules on particle surfaces can sometimes interfere with the sensing functions and reduce their efficacy. Janus particles with two separated faces and properties may provide elegant alternatives to decouple binding and sensing functions for these biosensors.

Han et al developed retroreflective Janus microparticles (RJPs) as a novel optical immunosensing probe for use in a nonspectroscopic retroreflection-based immunoassay. ${ }^{105}$ The RJPs consisted of silica particles, which were coated hemispherically with thin layers of aluminum and gold sequentially to produce highly reflective surfaces (Figure 18A). The gold outer layer was used to functionalize reacting antibodies on to particle surfaces. Once the antibodies bind to a substrate, 
A

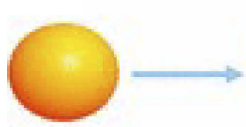

$\mathrm{Au}$

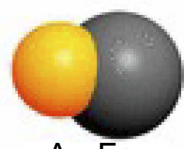

$\mathrm{Au}-\mathrm{Fe}$
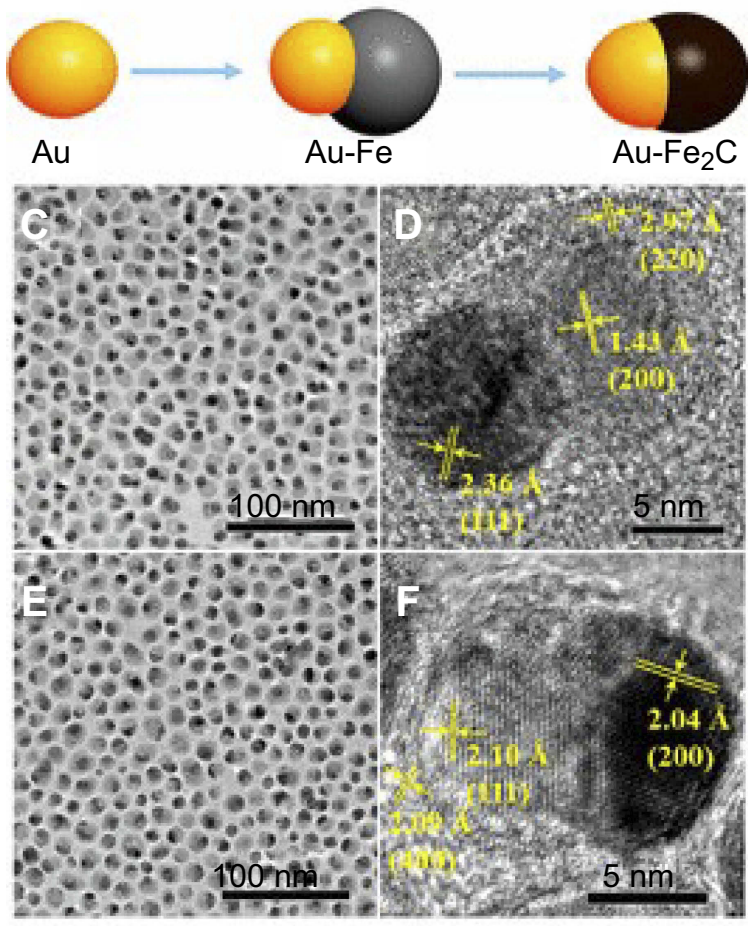

B
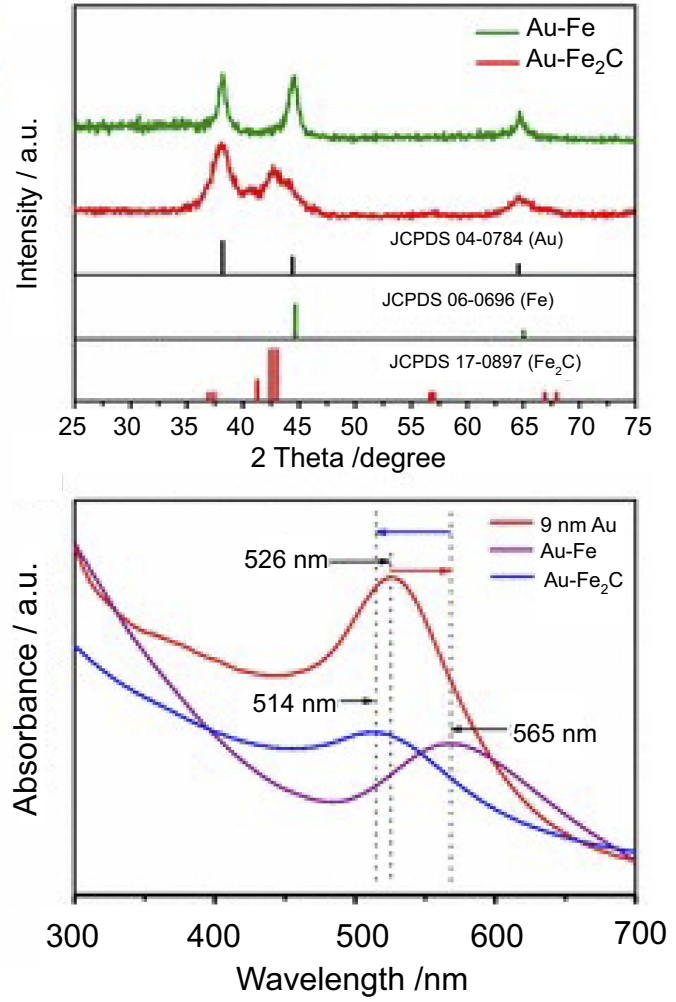

Figure 17 (A) Schematic depiction of the synthetic process of Au@Fe $\mathrm{F}_{2} \mathrm{C}$ janus NPs. (B) XRD patterns of Au@Fe heterostructures and Au@Fe 2 C Janus NPs. (C) TEM and (D) HRTEM images of Au@Fe heterostructures. (E) TEM and (F) HRTEM images of Au@Fe 2 Janus NPs. (G) UV-vis absorption spectra from 300 to 700 nm of 9 nm Au $\mathrm{NPs}, \mathrm{Au} @ \mathrm{Fe}$ heterostructures, and $\mathrm{Au} @ \mathrm{Fe}_{2} \mathrm{C}$ Janus NPs in hexane, respectively. Reprinted with permission from Ju Y, Zhang $\mathrm{H}$, Yu J, et al. Monodisperse Au-Fe $2 \mathrm{C}$ Janus nanoparticles: an attractive multifunctional material for triple-modal imaging-guided tumor photothermal therapy. ACS Nano. 20I7;।I(9):9239-9248. ${ }^{27}$ Copyright 20I7 American Chemical Society.

the RJPs were able to provide bright retroreflection signals which were easily detected using polychromatic white lightemitting diode (LED) irradiation (Figure 18B and C). The same group demonstrated the tunability of these Janus particles by conjugating streptavidin instead of antibodies to the gold surface of the RJPs (Figure 18D). Using these Janus particles, they showed the ability to detect $\mathrm{Hg}^{2+}$ in water. ${ }^{106}$ The study used two oligonucleotide probes, a stem-loop DNA probe and an assistant DNA probe (Figure 18E). When the mercury ions are absent, the assistant DNA probe does not hybridize with the stem-loop probe due to their thymine-thymine (T-T) mismatch. The surface-immobilized stem-loop DNA probe, therefore, remains a closed hairpin structure. When the $\mathrm{Hg}^{2+}$ ions are present, the DNA forms a double-stranded structure with the loop region via $\mathrm{Hg}^{2+}$-mediated T-T stabilization. This DNA hybridization induces stretching of the stem-loop DNA probe, exposing biotin. Streptavidin-modified RJPs are used as the optical signaling label to recognize the exposed biotin. The limit of $\mathrm{Hg}^{2+}$ detection of the system was recorded at $0.027 \mathrm{nM}$.
Similarly, Zhou et al created gold/polystyrene Janus particles by using the directional coating method. These Janus particles with one hydrophilic and one hydrophobic side were developed as an electrochemical sensor for the determination of ractopamine (RAC). ${ }^{107}$ RAC is a $\beta$ agonists, which can be found accumulated in livestock and may pose risks to the human cardiovascular and central nervous system. For this sensor, the hydrophobic part of the Janus particle was immobilized onto the surface of electrode. The hydrophilic part consists of Au NPs to which an aptamer that bound with RAC subsequently attached. The electrochemical sensor fabrication process is shown in Figure 19. The RAC aptamer-modified electrode could produce a well-defined differential pulse voltammetry signal as depicted in Figure 19 (stage "a"). When RAC was present, the interaction of RAC with the aptamer resulted in the hinder of the electron transition from the electrode surface. This change was observed in a decreased peak current of sensor (Figure 19, stage "b"). The detection limit of this electrochemical sensor was $3.3 \times 10^{-14} \mathrm{~mol} / \mathrm{L}$, which was 

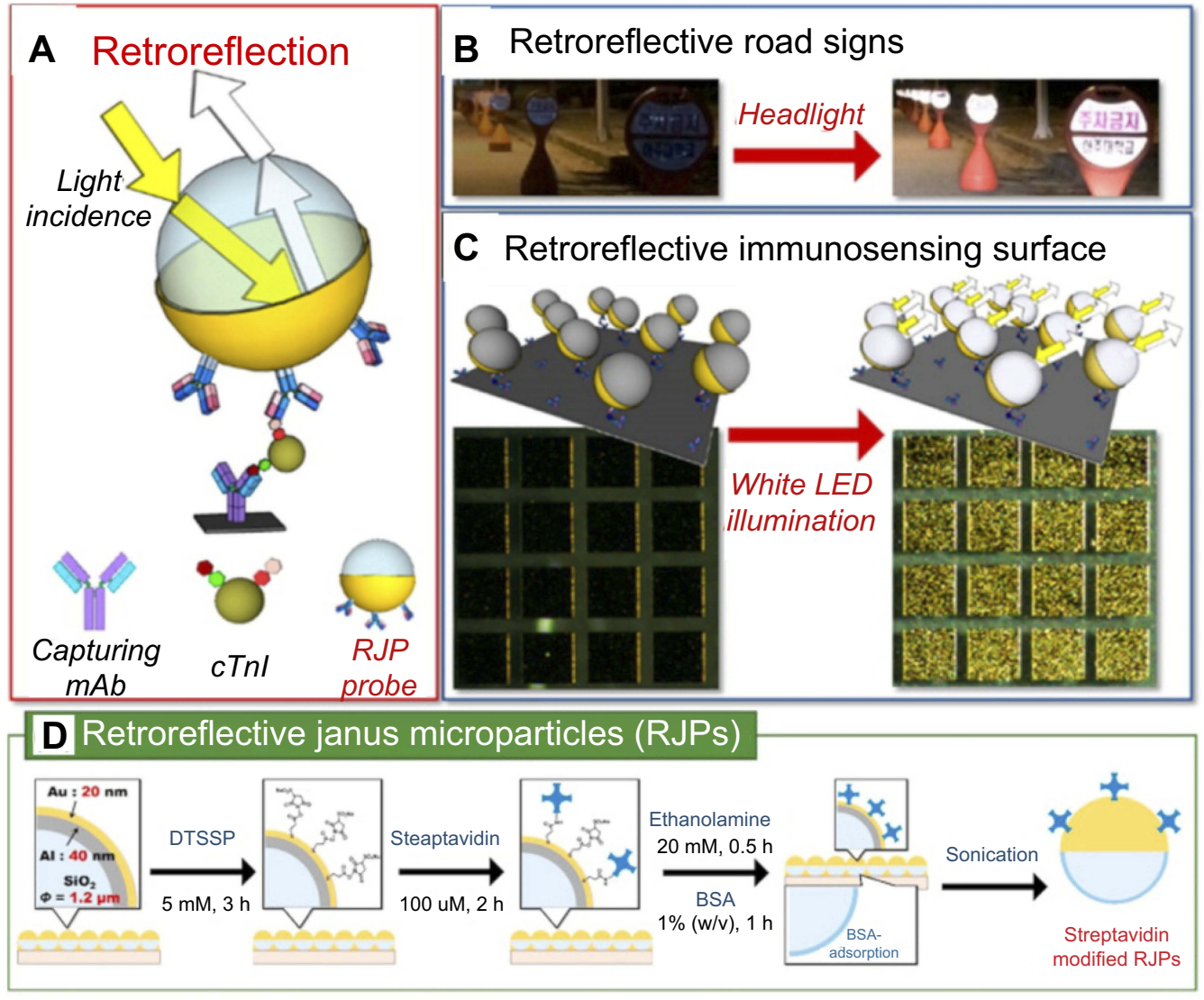

\section{E RJPs quantifying chip (RQC)}
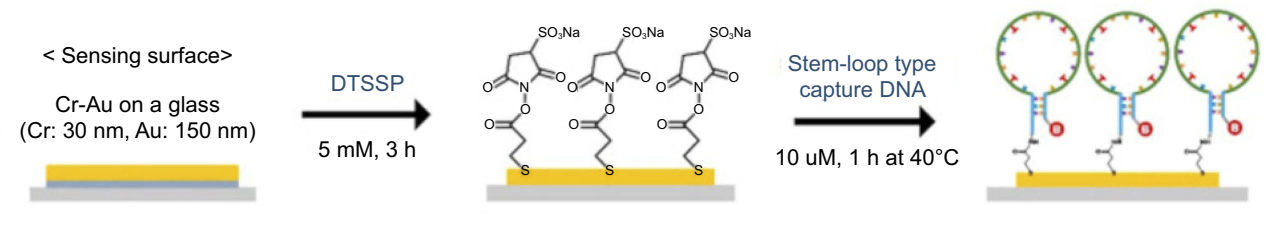

Figure 18 (A) Schematic diagram for the strategy of the proposed retroreflective cardiac troponin I (cTnl) sandwich immunoassay using RJP as an optical immunosensing probe. (B) Retroreflective road signs at night time. (C) Schematic illustrations and figures of retroreflective immunosensing surface before (left) and after (right) the white LED illumination. (D) Workflows of the functionalization procedures for streptavidin-modified retroreflective janus particles and (E) the construction process of $\mathrm{Hg}^{2+}$ recognition layer on the gold-modified surface of retroreflective quantifying chips. Reprinted with permission from Han YD, Kim H-S, Park YM, Chun HJ, Kim J-H, Yoon $\mathrm{HC}$. Retroreflective Janus microparticle as a nonspectroscopic optical immunosensing probe. ACS Appl Mater Interfaces. 2016;8(I7): 10767-10774.). Copyright 2016 American Chemical Society. ${ }^{105}$ and Chun HJ, Kim S, Han YD, et al. Water-soluble mercury ion sensing based on the thymine- $\mathrm{Hg}^{2+}$-thymine base pair using retroreflective 1490 janus particle as an optical signaling probe. Biosens Bioelectron. 2018;104:138-144. Copyright @ 2018 Elsevier B.V. ${ }^{106}$

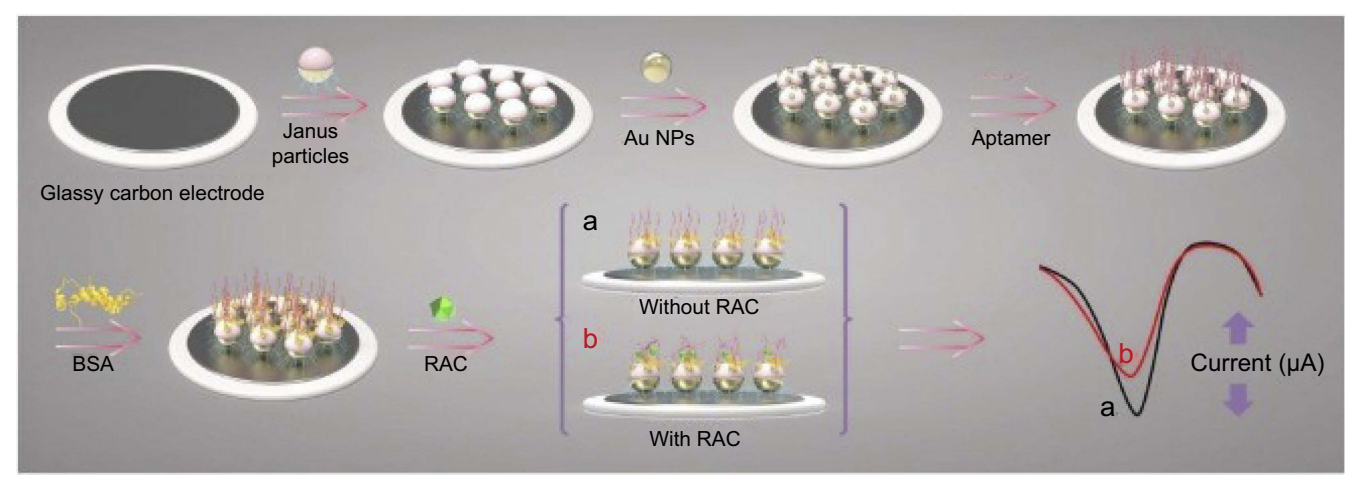

Figure 19 Schematic illustration of the electrochemical sensor fabrication process for the detection of ractopamine (RAC). The detection relies on the change in peak current of the sensor in the absence (A) and the presence (B) of RAC. Reprint with permission from Zhou Y, Yang YJ, Deng X, et al. Electrochemical sensor for determination of ractopamine based on aptamer/octadecanethiol Janus particles. Sens Actuat B Chem. 2018;276:204-210. ${ }^{107}$ Copyright (C) 20I8 Elsevier B.V. 
lower than most of existing methods such as fluorescence assay, HPLC-MS, and other types of electrochemical sensors. The proposed electrochemical sensor can be successfully applied to the determination of RAC in spiked human urine samples with good stability and reproducibility.

Biji and Patnaik studied Janus gold nanoclusters for sensing dopamine, an important neurotransmitter in the mammalian central nervous system. ${ }^{108}$ The Janus character was attributed in terms of ligand asymmetry and distribution. The subphase L-tryptophan acted as the reductant and capping agent at the lower hemisphere. Meanwhile, the hydrophobic tetraoctylammonium ion $\left(\mathrm{TOA}^{+}\right)$protected the upper hemisphere of the clusters at the air-water interface. The Janus scheme for the in situ formed clusters is illustrated in Figure 20A. The Janus Au nanocluster was used to modify a glassy carbon electrode which consequently transferred to different surface pressures/ cluster phase states, as a response to the electro-oxidation of dopamine. The sensitivity of dopamine detection depended on the $2 \mathrm{D}$ phase state, assembly, and organization of the Janus monolayer cluster. These also controlled the electronic communication between the clusters as a function of the intercluster distance, size, and orientation, ultimately influencing the electrocatalytic oxidation of the bio-analyte with improved sensitivity and detection limit. The study suggested that the noncovalent nature of the ligands on the core metal clusters facilitated the overall electro-catalytic oxidation of dopamine, as shown in Figure 20B. It was found that directed Brownian motion of the spherically charged clusters dictated by the $2 \mathrm{D}$ interfacial surface pressure was the driving force for the characteristic Janus cluster assembly into 1D Janus chains with varied dopamine detection efficiency.

\section{Janus micro- and nano-motors for biosensing}

Miniaturizing biosensors have attracted much attention from the research community due to the potential to improve their sensitivity and portability, while reducing cost and material requirements. However, a slow response time, the time it takes for the sensor to capture a detectable number of analyte

\section{A}

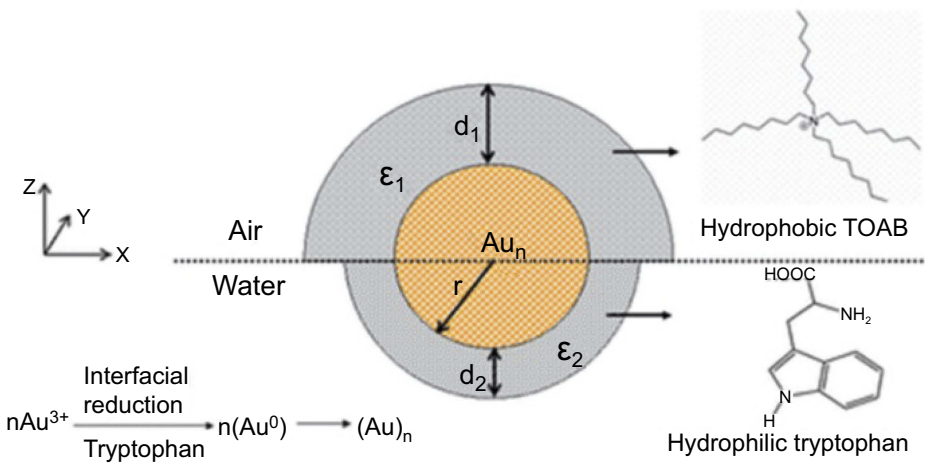

B

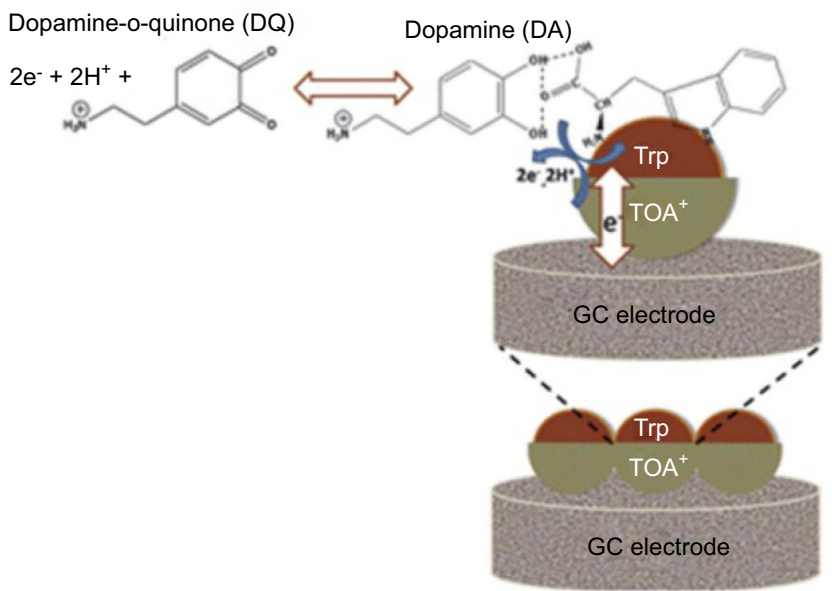

Figure 20 (A) Janus structure of the gold nanoclusters formed at the air-water interface. (B) Schematic representation of L-tryptophan-dopamine H-bonding interaction leading to electrocatalytic oxidation of dopamine at the Janus electrode/electrolyte interface. The blue arrows represent the redox reaction of dopamine at the gold nanocluster surface. The white arrow shows the electronic communication between the Janus cluster mediators to the glassy carbon electrode. Reprinted with permission from Biji P, Patnaik A. Interfacial Janus gold nanoclusters as excellent phase- and orientation-specific dopamine sensors. Analyst. 2012;137(20):4795-480 I. ${ }^{108}$ Copyright 2012 Royal Society of Chemistry. 
molecules, is a trade-off for the improved signal-to-noise ratio. ${ }^{109}$ One of the strategies to reduce response time involves self-propelled micro/nanomotors replenish the analyte-depleted solution. ${ }^{110,111}$ These micro/nanomotors could be propelled by gases generated from metal-catalyzed electrochemical reactions of toxic $\left(\mathrm{H}_{2} \mathrm{O}_{2}\right)^{112}$ or biofriendly (glucose or urea) fuels. As such, they are usually Janus particles with hemispherical metallic caps. There are also fuel-free micro/nanomotors ${ }^{113,114}$ which are powered by external stimuli such as magnetic, electric, or ultrasonic fields. ${ }^{115}$ An example of the nanomotors for therapeutic application has been briefly mentioned in Section "Janus particles for therapeutics". The micro/nano motor-based biosensors can be prepared easily by functionalizing the motors with specific bioreceptors. They can have different shapes and compositions but are usually asymmetric nanowire and microsphere and conical tube-based motors. ${ }^{115}$

Pacheco et al reported self-propelled microsensors for detecting lipopolysaccharides (LPS) from Salmonella enterica. ${ }^{116}$ These sensors relied on the simultaneous encapsulation of platinum NPs for enhanced bubblepropulsion and receptor-functionalized graphene quantum dots (GQDs) for selective binding with the target endotoxin (Figure 21). The GQDs interacted with the target endotoxins, which were LPS from Salmonella enterica in this case, and induce a rapid quenching of the native fluorescence of the micromotors. The sensors were able to detect concentrations as low as $0.07 \mathrm{ng} \mathrm{mL}^{-1}$ of endotoxin, which was well below the level toxic to humans $\left(275 \mu \mathrm{g} \mathrm{mL}^{-1}\right)$. Additionally, the sensors were shown to be stable for up to 2 months in an opaque vial without changes in their properties. The research group also reported magnetocatalytic Janus micromotors by adding iron oxide NPs to the platinum NP side of the Janus motors. The motors, therefore, were propelled in the presence of $\mathrm{H}_{2} \mathrm{O}_{2}$ or magnetic actuation. ${ }^{117}$

The use of Janus nanomotors to detect DNA and RNA has been reported by $\mathrm{Wu}$ et al. ${ }^{118}$ The motion-driven DNAsensing concept relied on measuring changes in the speed of unmodified catalytic Au@Pt Janus nanomotors induced by the dissolution of silver NP tags captured in a sandwich DNA hybridization assay. For this purpose, a gold electrode was first modified with a thiolated capture probe (SH-CP), mercaptohexanol (MCH), and dithiothreitol (DTT) (Figure 22). The thiolated DNA capture probe was able to form duplex with a complementary nucleic acid target and a silver nanoparticle-tagged detector probe (Figure 22A). The captured Ag NPs were dissolved using $\mathrm{H}_{2} \mathrm{O}_{2}$ to produce silver ions $\mathrm{Ag}^{+}$, which was proportionally related to the concentration of nucleic acid (Figure 22B). The authors observed that the $\mathrm{Au} @$ Pt Janus motors sped up in the presence of $\mathrm{Ag}^{+}$enriched fuel (Figure 22C). The travel distance of the nanomotor therefore could be used to quantify the amount of DNA
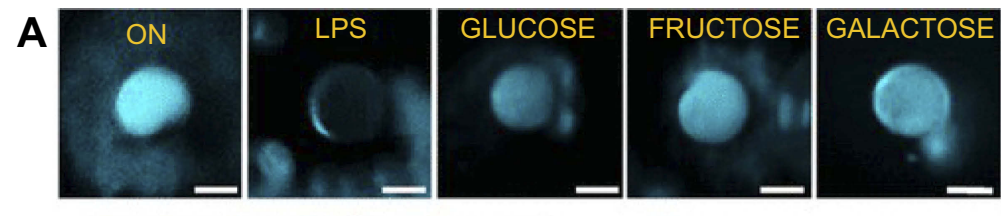

B

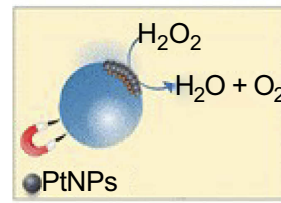

C
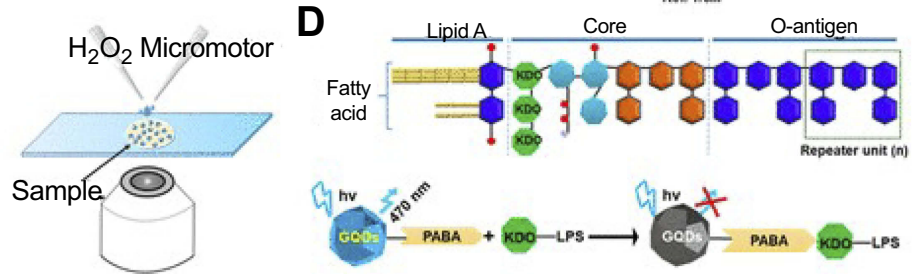

Figure 21 Self-propelled Janus microsensors for the detection of LPS from Salmonella enterica. (A) Real-time optical visualization of the LPS recognition event: time-lapse fluorescent images of the micromotors before (ON) and after LPS addition (OFF) and selectivity of the protocol in the presence of interfering saccharides. (B) Schematic of the microsensor operation and characterization. Optical images and Raman mapping showing the distribution of NPs, GQDs, and polycaprolactone (PCL) in the microsensor and corresponding Raman spectra. (C) Schematic of the setup for the Janus micromotors-based sensing protocol. (D) Schematics for structure of the LPS from Salmonella enterica and mechanism of quenching by LPS union to the GQDs recognition units. Experimental conditions: $15 \% \mathrm{H}_{2} \mathrm{O}_{2}$, surfactant, $5 \%$ (w/v) sodium cholate. Scale bars, $20 \mu \mathrm{m}$. Reprinted with permission from Pacheco M, Jurado-Sanchez B, Escarpa A. Sensitive monitoring of enterobacterial contamination of food using self-propelled Janus microsensors. Anal Chem. 2018;90(4):2912-2917. ${ }^{116}$ Copyright 2018 American Chemical Society. 


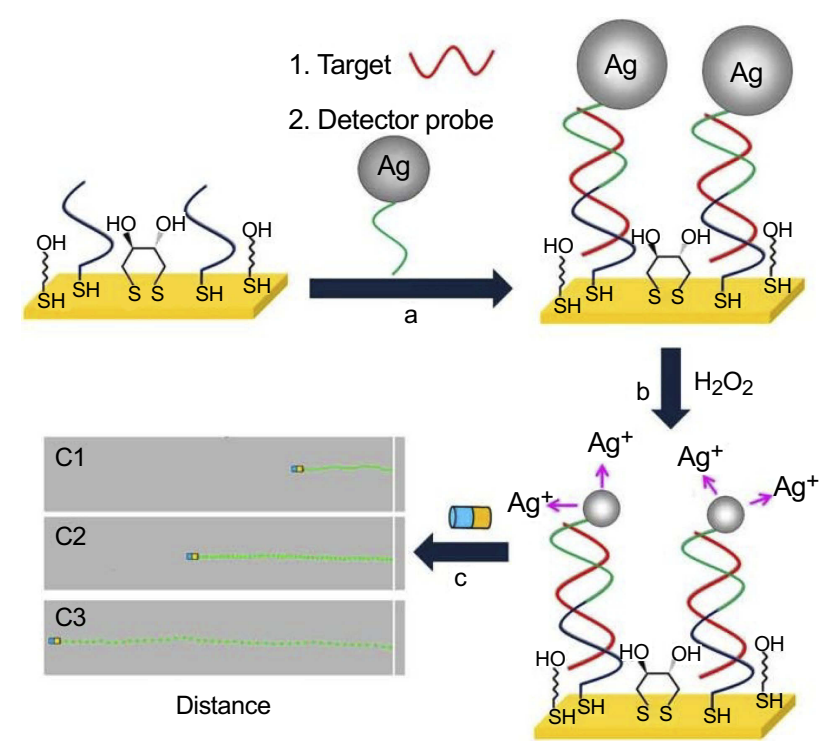

Figure 22 DNA detection based on silver ion-enhanced self-propulsion of Au@Pt nanomotors. (A) Hybridization of the target and capture of the Ag NP-tagged detector probe. (B) Dissolution of $\mathrm{Ag} \mathrm{NP}$ tags in $\mathrm{H}_{2} \mathrm{O}_{2}$, leading to $\mathrm{Ag}+$-enriched fuel. (C) Visual detection of the motion of the catalytic Au@Pt nanowire motors in the resulting $\mathrm{Ag}^{+}$-enriched fuel. Optical images superimposed with track lines show the distance traveled by the nanomotors in $\mathrm{H}_{2} \mathrm{O}_{2}$ fuel after DNA hybridization. Reprinted with permission from Wu J, Balasubramanian S, Kagan D, Manesh KM, Campuzano S, Wang J. Motion-based DNA detection using catalytic nanomotors. Nat Commun. 2010;1.Copyright (C) 2010, Springer Nature. ${ }^{118}$ target. For demonstration, the study showed that DNA and Escherichia coli 16S rRNA could be measured down to the attomole level and around $2000 \mathrm{CFU} / \mu \mathrm{L}$ level, respectively.

Janus motors have also been demonstrated as active SERS probes for remote, light-controlled, biochemical sensors by Wang et al. ${ }^{119}$ This matchlike one-dimensional sensor comprised a silica-coated silver nanowire head and a rough spherical $\mathrm{AgCl}$ tail (Figure 23). The motors worked as SERS probes based on the shell-isolated enhanced Raman mechanism. The $\mathrm{AgCl}$ tail served as photocatalytic nanoengine to provide a selfpropulsion force by light-induced self-diffusiophoresis through the decomposition of $\mathrm{AgCl}$. The phototactic behavior was utilized to achieve enrichment of the nanomotor-based SERS probes for on-demand biochemical sensing.

\section{Conclusions and perspectives}

In this review, we discussed the recent effort to apply Janus particles for effective delivery of therapeutics, imaging, and sensing in biological systems. Janus particles have two distinguished compartments on the two sides, usually made of materials with incompatible surfaces or chemistries such as hydrophilic versus hydrophobic. This compartmentalization

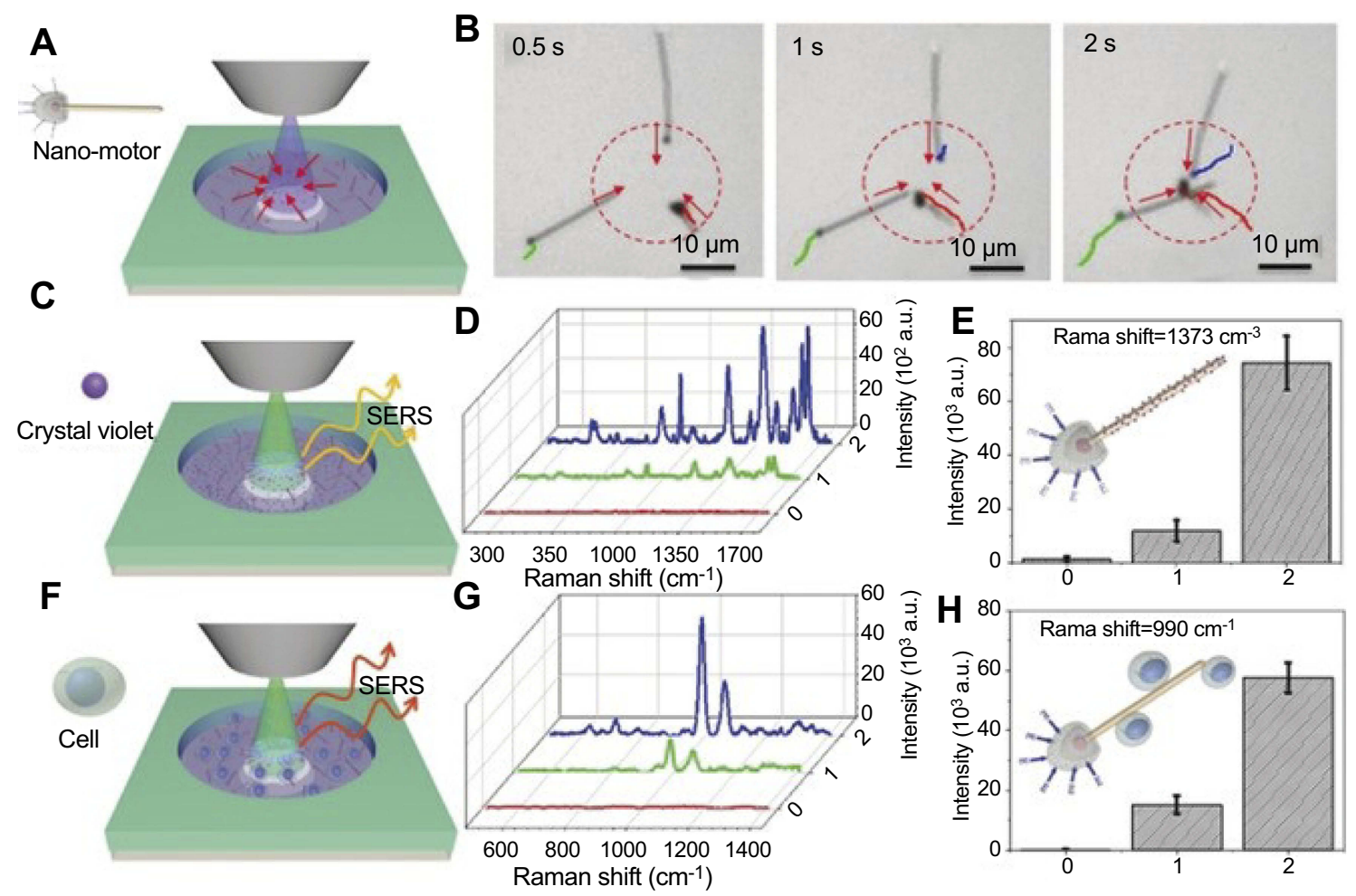

Figure 23 The light-guided biochemical SERS sensing by the phototactic nanomotor. (A) The schematic illustration and (B) video snapshots showing phototactic behavior of the nanomotors. Schematic illustrations of SERS sensing of crystal violet (CV) (C) and MCF-7 breast cancer cells (F). Raman spectra and intensity change of characteristic peaks of CV (D and E) and MCF-7 cells ( $\mathbf{G}$ and $\mathbf{H})$ under different conditions: (0) without any nanomotor; with nanomotor before (I) and after (2) focused UV-light irradiation for about $10 \mathrm{~s}$. Reprinted with permission from Wang Y, Zhou C, Wang W, et al. Photocatalytically powered matchlike nanomotor for light-guided active SERS sensing. Angew Chem Int Edit. 2018;57(40):13110-13113. Copyright (C) 2018 WILEY-VCH Verlag GmbH \& Co. KGaA, Weinheim. ${ }^{119}$ 
within a single Janus particle allows different properties to be available simultaneously and independently. For this reason, Janus particles have found applications in increasing number of nanomedicine areas, from dual-release drug delivery, to theranostics, and biosensing. This is particularly true in the last five years when many more Janus particles were tested in vitro and in vivo, providing large amount of valuable information regarding their behavior in biological systems. This is the direct result from an intense period of fundamental research involving the development of synthesis strategies of different types of Janus particles. However, there are still many challenges for Janus particles to overcome to reach their potential as nanomedicine. In our opinion, these include:

\section{Simpler and scale up production of Janus NPs}

As has been discussed previously, there are not many methods to produce Janus NPs reliably. Among the commonly used methods to produce Janus particles, microfluidic-based and electro co-jetting method can create highquality micron and sub-micron size Janus particles but difficult scale up. Similarly, directional coating method, in which a second component is deposited on a homogeneous monolayer of particles on a flat surface, can produce highquality Janus particles but not in large quantity. In contrary, the emulsion-based synthesis is easier to scale up, low cost, and applicable for a wider range of materials including organic, inorganic, and hybrid materials. However, this method has only been able to generate submicron and micron size Janus particles. In order to produce ultra-small Janus NPs, there are generally only two methods: block copolymer self-assembly for polymeric Janus NPs and anisotropic growth for nanodumbbell inorganic Janus NPs. For biomedical application, these NPs are usually required to go through further functionalization or drug loading processes. This is evidence from several studies mentioned in this review, in which multifunctional Janus NPs were produced through fairly complicated multi-step processes, thus limiting their yield and the possibility of scaling up. Additionally, since Janus NPs combine multiple materials and functionalities together, the theoretical compositional space, in which each component or property can be adjusted, becomes very large. To explore and manipulating each parameter to gain control over the NPs' in vivo properties, it requires the production of large material libraries, which are not possible using our current synthesis methods. More efficient high throughput formulation or synthesis approaches together with rapid screening characterization techniques and data analysis will need to be developed in the future.

\section{Refined characterisation techniques for Janus NPs}

Due to their unique anisotropic structure, Janus NPs are also difficult to characterize. Conventional techniques used in pharmaceutical and nanotechnology research such as dynamic light scattering, electrophoretic mobility, and mass spectrometry may provide general information but are not able to confirm anisotropy of the NPs. Traditionally, TEM, cryo-TEM, and occasionally SEM have been the main techniques to gain direct evidence of the Janus morphology. However, for this, the compartments are required to have sufficiently different electron densities, which may not be achievable for many polymers. Additionally, the particles must be relatively small and homogeneous for the TEM assessments to be accurate and representative. Alternatively, small angle X-ray scattering and neutron scattering, 2D NOESY NMR, and Forster resonance energy transfer (FRET) have been utilized to obtain indirect evidence of anisotropic within the produced Janus particles. In any case, a combination of several techniques is required to accurately characterize Janus NPs. As mentioned earlier, highthroughput characterization techniques should be developed and will provide benefit to the optimization process of Janus particles for biomedical applications.

3. Designing and testing in vitro and in vivo behaviors of Janus NPs

Even with the recent surge in developing Janus NPs for therapeutic, imaging, and sensing, our understanding of how these NPs behave in vitro and in vivo is still lacking. From the application point of view, recent studies have demonstrated the beneficial aspects of Janus NPs as drug delivery or imaging systems. The NPs can be meticulously engineered to encapsulate and release multiple drugs, to respond to multiple stimuli, and to provide imaging capability simultaneously with therapy. However, due to the lack of properly designed controls, the available data have not been always able to show the true advantages of using Janus particles over simply mixing components together or multi-component uniform core-shell particles. This should be an important consideration point for future studies, especially because it is much easier to alter component ratios in a simple mixture than in Janus particles. At a fundamental level, there are still unanswered questions regarding the mechanisms of their interaction with cells and 
proteins and their structural integrity in biological conditions. The anisotropic surface structure of Janus particles provides opportunities to incorporate multiple functionalities, but more complex interactions with biological systems are also expected.

Compared with the exploration of Janus particles for therapeutics and imaging, progress in their biosensing applications is still in early stages with even less in vivo information. Yet, promising proof-of-concept results have been demonstrated. It is clear that catalytic micro/nanomotors are useful for in vitro detection since peroxide fuel can be toxic to cells. Unless more biocompatible catalytic fuels can be developed, magnetic and ultrasound-propelled micro/nanomotors are ideal candidates for in vivo detection schemes.

One approach that may advance our understanding of Janus particles behavior in vitro and in vivo is the use of computer simulation, in which Janus particles of any size and shape can be created without too much trouble. ${ }^{120}$ Assessing Janus particles experimentally in conjunction with simulation may facilitate the realization of these particles as nanomedicine.

It should be noted that there are a vast number of anisotropic NPs in the literature, particularly metal@metal oxide NPs, which have been synthesized but not tested in vitro and in vivo. Similarly, many of the nanoparticles listed in the therapeutics section, for example, the Au@MS NPs developed by Cao et al in Section "Janus particles for in vivo imaging" can be also tested for theranostics to further expand their applications. Despite the limited number, reported studies have shown the exciting new use of these particles such as self-propelled Janus motors as active sensors, multicompartment Janus particles for carrying drugs of different water solubility, magnetic or fluorescent particles for effective imaging and diagnostics. More intense studies on the development of these particles for biomedical applications are expected in the near future.

\section{Acknowledgment}

TCL and NT are supported by the RMIT Vice Chancellor's Fellowships.

\section{Disclosure}

The authors report no conflicts of interest in this work.

\section{References}

1. Agrahari V, Agrahari V, Mitra AK. Nanocarrier fabrication and macromolecule drug delivery: challenges and opportunities. Ther Deliv. 2016;7(4):257-278. doi:10.4155/tde-2015-0012

2. Shi J, Kantoff PW, Wooster R, Farokhzad OC. Cancer nanomedicine: progress, challenges and opportunities. Nat Rev Cancer. 2017;17 (1):20-37. doi:10.1038/nrc.2016.108
3. Min Y, Caster JM, Eblan MJ, Wang AZ. Clinical translation of nanomedicine. Chem Rev. 2015;115(19):11147-11190. doi:10.1021/ acs.chemrev.5b00116

4. Zhai J, Luwor RB, Ahmed N, et al. Paclitaxel-loaded self-assembled lipid nanoparticles as targeted drug delivery systems for the treatment of aggressive ovarian cancer. ACS Appl Mater Interfaces. 2018;10 (30):25174-25185. doi:10.1021/acsami.8b08125

5. Li TJ, Huang CC, Ruan PW, et al. In vivo anti-cancer efficacy of magnetite nanocrystal - based system using locoregional hyperthermia combined with 5-fluorouracil chemotherapy. Biomaterials. 2013;34 (32):7873-7883. doi:10.1016/j.biomaterials.2013.07.012

6. Deng ZJ, Morton SW, Ben-Akiva E, Dreaden EC, Shopsowitz KE, Hammond PT. Layer-by-layer nanoparticles for systemic codelivery of an anticancer drug and siRNA for potential triple-negative breast cancer treatment. ACS Nano. 2013;7(11):9571-9584. doi: $10.1021 / \mathrm{nn} 4047925$

7. Tran N, Bye N, Moffat BA, et al. Dual-modality NIRF-MRI cubosomes and hexosomes: high throughput formulation and in vivo biodistribution. Mater Sci Eng C. 2017;71:584-593. doi:10.1016/j. msec.2016.10.028

8. Ho D, Sun X, Sun S. Monodisperse magnetic nanoparticles for theranostic applications. Acc Chem Res. 2011;44(10):875-882. doi:10.1021/ar200090c

9. Feng L, Mumper RJ. A critical review of lipid-based nanoparticles for taxane delivery. Cancer Lett. 2013;334(2):157-175. doi:10.1016/j.canlet.2012.07.006

10. Danhier F, Ansorena E, Silva JM, Coco R, Le Breton A, Preat V. PLGAbased nanoparticles: an overview of biomedical applications. J Control Release. 2012;161(2):505-522. doi:10.1016/j.jconrel.2012.01.043

11. Sharma A, Goyal AK, Rath G. Recent advances in metal nanoparticles in cancer therapy. $J$ Drug Target. 2018;26(8):617-632. doi:10.1080/1061186X.2017.1400553

12. Mulet X, Boyd BJ, Drummond CJ. Advances in drug delivery and medical imaging using colloidal lyotropic liquid crystalline dispersions. J Colloid Interface Sci. 2013;393:1-20. doi:10.1016/j.jcis.2012.10.014

13. Granick S, Jiang S, Chen Q. Janus particles. Phys Today. 2009;62 (7):68-69. doi:10.1063/1.3177238

14. Kaewsaneha C, Tangboriboonrat P, Polpanich D, Eissa M, Elaissari A. Janus colloidal particles: preparation, properties, and biomedical applications. ACS Appl Mater Interfaces. 2013;5 (6): 1857-1869. doi:10.1021/am302528g

15. Du J, O'Reilly RK. Anisotropic particles with patchy, multicompartment and Janus architectures: preparation and application. Chem Soc Rev. 2011;40(5):2402-2416. doi:10.1039/c0cs00216j

16. Casagrande C, Fabre P, Raphaël E, Veyssié M. "Janus Beads": realization and behaviour at water/oil interfaces. EPL Europhys Lett. 1989;9(3):251. doi:10.1209/0295-5075/9/3/011

17. de Gennes PG. Soft matter. Science. 1992;256(5056):495. doi:10.1126/science. 256.5056 .495

18. Liang FX, Zhang CL, Yang ZZ. Rational design and synthesis of Janus composites. Adv Mater. 2014;26(40):6944-6949. doi:10.1002/adma.201305415

19. Walther A, MüLler AH. Janus particles: synthesis, self-assembly, physical properties, and applications. Chem Rev. 2013;113 (7):5194-5261. doi:10.1021/cr300089t

20. Kim D, Yu MK, Lee TS, Park JJ, Jeong YY, Jon S. Amphiphilic polymer-coated hybrid nanoparticles as CT/MRI dual contrast agents. Nanotechnology. 2011;22(15):155101. doi:10.1088/0957-4484/22/15/ 155101

21. Schick I, Lorenz S, Gehrig D, et al. Inorganic Janus particles for biomedical applications. Beilstein J Nanotechnol. 2014;5 (1):2346-2362. doi:10.3762/bjnano.5.244

22. Tran N, Mulet X, Hawley AM, et al. First direct observation of stable internally ordered janus nanoparticles created by lipid self-assembly. Nano Lett. 2015;15(6):4229-4233. doi:10.1021/acs. nanolett.5b01751 
23. Garbuzenko OB, Winkler J, Tomassone MS, Minko T. Biodegradable Janus nanoparticles for local pulmonary delivery of hydrophilic and hydrophobic molecules to the lungs. Langmuir. 2014;30 (43):12941-12949. doi:10.1021/la502144z

24. Hayes OG, McMillan JR, Lee B, Mirkin CA. DNA-encoded protein Janus nanoparticles. $J$ Am Chem Soc. 2018;140 (29):9269-9274. doi:10.1021/jacs.8b05640

25. Cao H, Yang Y, Chen X, Shao Z. Intelligent Janus nanoparticles for intracellular real-time monitoring of dual drug release. Nanoscale. 2016;8(12):6754-6760. doi:10.1039/c6nr00987e

26. Lee K, Zhang L, Yi Y, Wang X, Yu Y. Rupture of Lipid Membranes Induced by Amphiphilic Janus Nanoparticles. ACS Nano. 2018;12 (4):3646-3657. doi:10.1021/acsnano.8b00759

27. Ju Y, Zhang H, Yu J, et al. Monodisperse Au-fe2C Janus nanoparticles: an attractive multifunctional material for triple-modal imaging-guided tumor photothermal therapy. ACS Nano. 2017;11 (9):9239-9248. doi:10.1021/acsnano.7b04461

28. Walther A, Müller AH. Janus particles. Soft Matter. 2008;4 (4):663-668. doi:10.1039/b718131k

29. Lattuada M, Hatton TA. Synthesis, properties and applications of Janus nanoparticles. Nano Today. 2011;6(3):286-308. doi:10.1016/ j.nantod.2011.04.008

30. Tran L-T-C, Lesieur S, Faivre V. Janus nanoparticles: materials, preparation and recent advances in drug delivery. Expert Opin Drug Deliv. 2014;11(7):1061-1074. doi:10.1517/17425247.2014.915806

31. Tao G, Bai Z, Chen Y, et al. Generic synthesis and versatile applications of molecularly organic-inorganic hybrid mesoporous organosilica nanoparticles with asymmetric Janus topologies and structures. Nano Res. 2017;10(11):3790-3810. doi:10.1007/ s12274-017-1592-5

32. Yi Y, Sanchez L, Gao Y, Yu Y. Janus particles for biological imaging and sensing. Analyst. 2016;141(12):3526-3539. doi: $10.1039 / \mathrm{c} 6 \mathrm{an} 00325 \mathrm{~g}$

33. Hwang S, Lahann J. Differentially degradable Janus particles for controlled release applications. Macromol Rapid Commun. 2012;33 (14):1178-1183. doi:10.1002/marc.201200054

34. Xie H, She Z-G, Wang S, Sharma G, Smith JW. One-step fabrication of polymeric Janus nanoparticles for drug delivery. Langmuir. 2012;28(9):4459-4463. doi:10.1021/la2042185

35. Adams JD, Flora K, Goldspiel B, Wilson J, Arbuck S, Finley R. Taxol: a history of pharmaceutical development and current pharmaceutical concerns. J Natl Cancer Inst Monogr. 1993;1993(15):141-147.

36. Singal PK, Iliskovic N. Doxorubicin-induced cardiomyopathy. $N$ Engl $J$ Med. 1998;339(13):900-905. doi:10.1056/ NEJM199809243391307

37. Lahann J. Recent progress in nano-biotechnology: compartmentalized micro-and nanoparticles via electrohydrodynamic co-jetting. Small. 2011;7(9):1149-1156. doi:10.1002/smll.201002002

38. Dehghani E, Salami-Kalajahi M, Roghani-Mamaqani H. Simultaneous two drugs release form Janus particles prepared via polymerization-induced phase separation approach. Colloids Surf B Biointerfaces. 2018;170:85-91. doi:10.1016/j.colsurfb.2018.05.067

39. Romanski FS, Winkler JS, Riccobene RC, Tomassone MS. Production and Characterization of Anisotropic Particles from Biodegradable Materials. Langmuir. 2012;28(8):3756-3765. doi:10.1021/la2044834

40. Rahmani S, Villa CH, Dishman AF, et al. Long-circulating Janus nanoparticles made by electrohydrodynamic co-jetting for systemic drug delivery applications. J Drug Target. 2015;23(7-8):750-758. doi:10.3109/1061186X.2015.1076428

41. Tran N, Hawley AM, Zhai J, et al. High-throughput screening of saturated fatty acid influence on nanostructure of lyotropic liquid crystalline lipid nanoparticles. Langmuir. 2016;32(18):4509-4520. doi:10.1021/acs.langmuir.5b03769
42. Tran N, Mulet X, Hawley AM, et al. Manipulating the ordered nanostructure of self-assembled monoolein and phytantriol nanoparticles with unsaturated fatty acids. Langmuir. 2018;34 (8):2764-2773. doi:10.1021/acs.langmuir.7b03541

43. Tran N, Hocquet M, Eon B, et al. Non-lamellar lyotropic liquid crystalline nanoparticles enhance the antibacterial effects of rifampicin against Staphylococcus aureus. J Colloid Interface Sci. 2018;519:107-118. doi:10.1016/j.jcis.2018.02.048

44. Zhai J, Scoble JA, Li N, et al. Epidermal growth factor receptor-targeted lipid nanoparticles retain self-assembled nanostructures and provide high specificity. Nanoscale. 2015;7 (7):2905-2913. doi:10.1039/c4nr05200e

45. Kim H, Cuboplexes: LC. Topologically active siRNA delivery. ACS Nano. 2015;9(10):10214-10226. doi:10.1021/acsnano.5b03902

46. Sarkar S, Tran N, Rashid MH, et al. Toward cell membrane biomimetic lipidic cubic phases: a high-throughput exploration of lipid compositional space. ACS Applied Bio Mater. 2018;2 (1):182-195. doi:10.1021/acsabm.8b00539

47. Boyd BJ, Whittaker DV, Khoo S-M, Davey G. Lyotropic liquid crystalline phases formed from glycerate surfactants as sustained release drug delivery systems. Int J Pharm. 2006;309(1-2):218-226. doi:10.1016/j.ijpharm.2005.11.033

48. Tran N, Mulet X, Hawley AM, et al. Nanostructure and cytotoxicity of self-assembled monoolein-capric acid lyotropic liquid crystalline nanoparticles. RSC Adv. 2015;5(34):26785-26795. doi:10.1039/C5RA02604K

49. Zhai J, Suryadinata R, Luan B, et al. Amphiphilic brush polymers produced using the RAFT polymerisation method stabilise and reduce the cell cytotoxicity of lipid lyotropic liquid crystalline nanoparticles. Faraday Discuss. 2016;191:545-563. doi:10.1039/c6fd00039h

50. Zhai J, Hinton TM, Waddington LJ, et al. Lipid-PEG conjugates sterically stabilize and reduce the toxicity of phytantriol-based lyotropic liquid crystalline nanoparticles. Langmuir. 2015;31 (39):10871-10880. doi:10.1021/acs.langmuir.5b02797

51. Wang C, Xu C. Design, synthesis and applications of dumbbell-like nanoparticles. In: Janus Particle Synthesis, Self-Assembly and Applications. Editors: Shan Jiang, Steve Granick. Publisher: Royal Society of Chemistry, Cambridge, UK; 2012:29-53.

52. Huang X, Jain PK, El-Sayed IH, El-Sayed MA. Plasmonic photothermal therapy (PPTT) using gold nanoparticles. Lasers Med Sci. 2008;23(3):217. doi:10.1007/s10103-007-0470-x

53. Pissuwan D, Cortie CH, Valenzuela SM, Cortie MB. Functionalised gold nanoparticles for controlling pathogenic bacteria. Trends Biotechnol. 2010;28(4):207-213. doi:10.1016/j.tibtech.2009.12.004

54. Elbakry A, Zaky A, Liebl R, Rachel R, Goepferich A, Breunig M. Layer-by-layer assembled gold nanoparticles for siRNA delivery. Nano Lett. 2009;9(5):2059-2064. doi:10.1021/n19003865

55. Hajipour MJ, Fromm KM, Ashkarran AA, et al. Antibacterial properties of nanoparticles. Trends Biotechnol. 2012;30 (10):499-511. doi:10.1016/j.tibtech.2012.06.004

56. Tran N, Tran PA. Nanomaterial-based treatments for medical device-associated infections. ChemPhysChem. 2012;13 (10):2481-2494. doi:10.1002/cphc.201200091

57. Laurent S, Dutz S, Häfeli UO, Mahmoudi M. Magnetic fluid hyperthermia: focus on superparamagnetic iron oxide nanoparticles. Adv Colloid Interface Sci. 2011;166(1-2):8-23. doi:10.1016/j.cis.2011.04.003

58. Tran N, Webster TJ. Magnetic nanoparticles: biomedical applications and challenges. J Mater Chem. 2010;20(40):8760-8767. doi:10.1039/c0jm00994f

59. Slowing II, Vivero-Escoto JL, Wu C-W, Lin VS-Y. Mesoporous silica nanoparticles as controlled release drug delivery and gene transfection carriers. Adv Drug Deliv Rev. 2008;60(11):1278-1288. doi:10.1016/j.addr.2008.03.012 
60. Wang L, Zhao W, Tan W. Bioconjugated silica nanoparticles: development and applications. Nano Res. 2008;1(2):99-115. doi:10.1007/s12274-008-8018-3

61. Wang C, Yin H, Dai S, Sun S. A general approach to noble metalmetal oxide dumbbell nanoparticles and their catalytic application for CO oxidation. Chem Mater. 2010;22(10):3277-3282. doi:10.1021/cm100603r

62. Buck MR, Bondi JF, Schaak RE. A total-synthesis framework for the construction of high-order colloidal hybrid nanoparticles. Nat Chem. 2012;4(1):37. doi:10.1038/nchem.1195

63. Gu H, Yang Z, Gao J, Chang C, Xu B. Heterodimers of nanoparticles: formation at a liquid- liquid interface and particle-specific surface modification by functional molecules. J Am Chem Soc. 2005;127(1):34-35. doi:10.1021/ja045220h

64. Gu H, Zheng $\mathrm{R}$, Zhang $\mathrm{X}, \mathrm{Xu}$ B. Facile one-pot synthesis of bifunctional heterodimers of nanoparticles: a conjugate of quantum dot and magnetic nanoparticles. J Am Chem Soc. 2004;126 (18):5664-5665. doi:10.1021/ja0496423

65. $\mathrm{Xu} \mathrm{C,} \mathrm{Xie} \mathrm{J,} \mathrm{Ho} \mathrm{D,} \mathrm{et} \mathrm{al.} \mathrm{Au-fe} 3 \mathrm{O} 4$ dumbbell nanoparticles as dual-functional probes. Angew Chem Int Ed. 2008;47(1):173-176. doi:10.1002/anie.200704392

66. Jishkariani D, Wu Y, Wang D, Liu Y, van Blaaderen A, Murray CB. Preparation and self-assembly of dendronized Janus Fe3O4-pt and Fe3O4-au heterodimers. ACS Nano. 2017;11(8):7958-7966. doi:10.1021/acsnano.7b02485

67. Lopes G, Vargas JM, Sharma SK, et al. Ag-Fe3O4 dimer colloidal nanoparticles: synthesis and enhancement of magnetic properties. J Phys Chem C. 2010;114(22):10148-10152. doi:10.1021/jp102311u

68. Nakhjavan B, Tahir MN, Natalio F, et al. Phase separated Cu@Fe3O4 heterodimer nanoparticles from organometallic reactants. J Mater Chem. 2011;21(24):8605-8611. doi:10.1039/c1jm10922g

69. Wang C, Daimon H, Sun S. Dumbbell-like Pt-Fe3O4 nanoparticles and their enhanced catalysis for oxygen reduction reaction. Nano Lett. 2009;9(4):1493-1496. doi:10.1021/n18034724

70. Nakhjavan B, Tahir MN, Natalio F, et al. Ni@Fe2O3 heterodimers: controlled synthesis and magnetically recyclable catalytic application for dehalogenation reactions. Nanoscale. 2012;4 (15):4571-4577. doi:10.1039/c2nr12121b

71. Schick I, Lorenz S, Gehrig D, et al. Multifunctional two-photon active silica-coated $\mathrm{Au} @$ MnO Janus particles for selective dual functionalization and imaging. $J$ Am Chem Soc. 2014;136 (6):2473-2483. doi:10.1021/ja410787u

72. Schladt TD, Graf T, Köhler O, et al. Synthesis and magnetic properties of FePt@MnO nano-heteroparticles. Chem Mater. 2012;24(3):525-535. doi:10.1021/cm2030685

73. Díez P, Sánchez A, Gamella M, et al. Toward the design of smart delivery systems controlled by integrated enzyme-based biocomputing ensembles. J Am Chem Soc. 2014;136(25):9116-9123. doi:10.1021/ja503578b

74. Chang Z-M, Wang Z, M-M L, et al. Janus silver mesoporous silica nanobullets with synergistic antibacterial functions. Colloids Surf B Biointerfaces. 2017;157:199-206. doi:10.1016/j. colsurfb.2017.05.079

75. Wang Z, Chang Z, Lu M, et al. Janus silver/silica nanoplatforms for light-activated liver cancer chemo/photothermal therapy. ACS Appl Mater Interfaces. 2017;9(36):30306-30317. doi:10.1021/ acsami.7b06446

76. Shao D, Li J, Zheng X, et al. Janus "nano-bullets" for magnetic targeting liver cancer chemotherapy. Biomaterials. 2016;100:118-133. doi:10.1016/j.biomaterials.2016.05.030

77. Wang Z, Wang Y, Chang Z, et al. Berberine-loaded Janus nanocarriers for magnetic field-enhanced therapy against hepatocellular carcinoma. Chem Biol Drug Des. 2017;89(3):464-469. doi:10.1111/cbdd. 12866
78. Knežević NŽ, Lin VS-Y. A magnetic mesoporous silica nanoparticle-based drug delivery system for photosensitive cooperative treatment of cancer with a mesopore-capping agent and mesopore-loaded drug. Nanoscale. 2013;5(4):1544-1551. doi:10.1039/c2nr33417h

79. Ma M, Chen H, Chen Y, et al. Au capped magnetic core/mesoporous silica shell nanoparticles for combined photothermo-/chemotherapy and multimodal imaging. Biomaterials. 2012;33 (3):989-998. doi:10.1016/j.biomaterials.2011.10.017

80. Zhang L, Qiao S, Jin Y, Chen Z, Gu H, Lu GQ. Magnetic hollow spheres of periodic mesoporous organosilica and Fe3O4 nanocrystals: fabrication and structure control. Adv Mater. 2008;20 (4):805-809. doi:10.1002/(ISSN)1521-4095

81. Li X, Zhou L, Wei Y, El-Toni AM, Zhang F, Zhao D. Anisotropic growth-induced synthesis of dual-compartment Janus mesoporous silica nanoparticles for bimodal triggered drugs delivery. $\mathrm{J} \mathrm{Am}$ Chem Soc. 2014;136(42):15086-15092. doi:10.1021/ja508733r

82. Xuan M, Shao J, Lin X, Dai L, He Q. Self-propelled janus mesoporous silica nanomotors with sub-100 nm diameters for drug encapsulation and delivery. ChemPhysChem. 2014;15 (11):2255-2260. doi:10.1002/cphc.201402111

83. Wu Y, Lin X, Wu Z, MöHwald H, He Q. Self-propelled polymer multilayer Janus capsules for effective drug delivery and light-triggered release. ACS Appl Mater Interfaces. 2014;6 (13):10476-10481. doi:10.1021/am502458h

84. Salem AK, Hung CF, Kim TW, Wu TC, Searson PC, Leong KW. Multi-component nanorods for vaccination applications. Nanotechnology. 2005;16(4):484. doi:10.1088/0957-4484/16/4/025

85. Salem AK, Searson PC, Leong KW. Multifunctional nanorods for gene delivery. Nat Mater. 2003;2(10):668. doi:10.1038/nmat974

86. Wang F, Pauletti GM, Wang J, et al. Dual surface-functionalized janus nanocomposites of polystyrene/Fe3O4@ $\mathrm{SiO} 2$ for simultaneous tumor cell targeting and stimulus-induced drug release. $A d v$ Mater. 2013;25(25):3485-3489. doi:10.1002/adma.201301376

87. Khoee S, Karimi MR. Dual-drug loaded Janus graphene oxide-based thermoresponsive nanoparticles for targeted therapy. Polymer. 2018;142:80-98. doi:10.1016/j.polymer.2018.03.022

88. Zhang L, Li S, Chen X. et al. Tailored surfaces on $2 \mathrm{D}$ material: UFO-like cyclodextrin-Pd nanosheet/metal organic framework Janus nanoparticles for synergistic cancer therapy. Adv Funct Mater;2018. 1803815. doi:10.1002/adfm.201803815

89. Guarrotxena N, García O, Quijada-Garrido I. Synthesis of Au@ polymer nanohybrids with transited core-shell morphology from concentric to eccentric Emoji-N or Janus nanoparticles. Sci Rep. 2018;8(1):5721. doi:10.1038/s41598-018-24078-8

90. Chen G, Gibson KJ, Liu D, et al. Regioselective surface encoding of nanoparticles for programmable self-assembly. Nat Mater. 2018;18:2.

91. Zhang L, Chen Y, Li Z, et al. Tailored Synthesis of octopus-type janus nanoparticles for synergistic actively-targeted and chemo-photothermal therapy. Angew Chem Int Ed. 2016;55(6):2118-2121. doi:10.1002/ anie. 201510409

92. Shaghaghi B, Khoee S, Bonakdar S. Preparation of multifunctional Janus nanoparticles on the basis of SPIONs as targeted drug delivery system. Int $J$ Pharm. 2019;559:1-12. doi:10.1016/j. ijpharm.2019.01.020

93. Reguera J, de Aberasturi DJ, Henriksen-Lacey M, et al. Janus plasmonic-magnetic gold-iron oxide nanoparticles as contrast agents for multimodal imaging. Nanoscale. 2017;9(27):9467-9480. doi:10.1039/ c7nr01406f

94. Liu Y, Yang X, Huang Z, et al. Magneto-plasmonic janus vesicles for magnetic field-enhanced photoacoustic and magnetic resonance imaging of tumors. Angew Chem Int Ed. 2016;55(49):15297-15300. doi:10.1002/anie. 201608338 
95. Shao D, Zhang X, Liu W, et al. Janus silver-mesoporous silica nanocarriers for SERS traceable and $\mathrm{pH}$-sensitive drug delivery in cancer therapy. ACS Appl Mater Interfaces. 2016;8(7):4303-4308. doi:10.1021/acsami.5b11310

96. Wang Y-S, Shao D, Zhang L, et al. Gold nanorods-silica Janus nanoparticles for theranostics. Appl Phys Lett. 2015;106 (17):173705. doi:10.1063/1.4919454

97. Wang Y-S, Dong W-F, Xia H, et al. One-pot preparation of novel asymmetric structure nanoparticles and its application in catalysis. RSC Adv. 2014;4(82):43586-43589. doi:10.1039/ C4RA05950F

98. Chen X, Li G, Han Q, et al. Rational design of branched Au-fe3O4 Janus nanoparticles for simultaneous trimodal imaging and photothermal therapy of cancer cells. Chem Eur J. 2017;23 (68):17204-17208. doi:10.1002/chem.201704514

99. Zhang Q, Zhang L, Li S, et al. Designed synthesis of Au/Fe3O4@ C Janus nanoparticles for dual-modal imaging and actively targeted chemo-photothermal synergistic therapy of cancer cells. Chem Eur J. 2017;23(68):17242-17248. doi:10.1002/chem.201703498

100. Zhang L, Zhang M, Zhou L, et al. Dual drug delivery and sequential release by amphiphilic Janus nanoparticles for liver cancer theranostics. Biomaterials. 2018;181:113-125. doi:10.1016/j. biomaterials.2018.07.060

101. Li S, Zhang L, Chen X, et al. Selective growth synthesis of ternary Janus nanoparticles for imaging-guided synergistic chemo- and photothermal therapy in the second NIR window. ACS Appl Mater Interfaces. 2018;10 (28):24137-24148. doi:10.1021/acsami.8b06527

102. Iqbal MZ, Ren W, Saeed M, et al. A facile fabrication route for binary transition metal oxide-based Janus nanoparticles for cancer theranostic applications. Nano Res. 2018;11(10):5735-5750. doi:10.1007/s12274-017-1628-x

103. Wang H, Li S, Zhang L, et al. Tunable fabrication of folic acid-Au@ poly (acrylic acid)/mesoporous calcium phosphate Janus nanoparticles for CT imaging and active-targeted chemotherapy of cancer cells. Nanoscale. 2017;9(38):14322-14326. doi:10.1039/c7nr05382g

104. Zhang M, Zhang L, Chen Y, Li L, Su Z, Wang C. Precise synthesis of unique polydopamine/mesoporous calcium phosphate hollow Janus nanoparticles for imaging-guided chemo-photothermal synergistic therapy. Chem Sci. 2017;8(12):8067-8077. doi:10.1039/c7sc03521g

105. Han YD, Kim H-S, Park YM, Chun HJ, Kim J-H, Yoon HC. Retroreflective Janus microparticle as a nonspectroscopic optical immunosensing probe. ACS Appl Mater Interfaces. 2016;8 (17):10767-10774. doi:10.1021/acsami.6b02014

106. Chun HJ, Kim S, Han YD, et al. Water-soluble mercury ion sensing based on the thymine-Hg2+-thymine base pair using retroreflective Janus particle as an optical signaling probe. Biosens Bioelectron. 2018;104:138-144. doi:10.1016/j.bios.2018.01.008
107. Zhou Y, Yang YJ, Deng X, et al. Electrochemical sensor for determination of ractopamine based on aptamer/octadecanethiol Janus particles. Sens Actuat B Chem. 2018;276:204-210. doi:10.1016/j.snb.2018.08.110

108. Biji P, Patnaik A. Interfacial Janus gold nanoclusters as excellent phase- and orientation-specific dopamine sensors. Analyst. 2012;137(20):4795-4801. doi:10.1039/c2an35964b

109. Soleymani L, Li F. Mechanistic challenges and advantages of biosensor miniaturization into the nanoscale. ACS Sens. 2017;2 (4):458-467. doi:10.1021/acssensors.7b00069

110. Jurado-Sánchez B. Nanoscale biosensors based on self-propelled objects. Biosensors. 2018;8(3):59. doi:10.3390/bios8030059

111. Campuzano S, de Avila BEF, Yanez-Sedeno P, Pingarron JM, Wang J. Nano/microvehicles for efficient delivery and (bio) sensing at the cellular level. Chem Sci. 2017;8(10):6750-6763. doi:10.1039/c7sc02434g

112. Balasubramanian S, Kagan D, Hu CMJ, et al. Micromachineenabled capture and isolation of cancer cells in complex media. Angew Chem Int Edit. 2011;50(18):4161-4164. doi:10.1002/ anie. 201100115

113. Campuzano S, Orozco J, Kagan D, et al. Bacterial isolation by lectin-modified microengines. Nano Lett. 2012;12(1):396-401. doi:10.1021/nl203717q

114. Moreno-Guzman M, Jodra A, Lopez MA, Escarpa A. Selfpropelled enzyme-based motors for smart mobile electrochemical and optical biosensing. Anal Chem. 2015;87(24):12380-12386. doi:10.1021/acs.analchem.5b03928

115. Yu XP, Wu J, Ju HX. The application of micro/nanomotor in biosensing. Prog Chem. 2014;26(10):1712-1719.

116. Pacheco M, Jurado-Sanchez B, Escarpa A. Sensitive monitoring of enterobacterial contamination of food using self-propelled Janus microsensors. Anal Chem. 2018;90(4):2912-2917. doi:10.1021/ acs.analchem. $7 \mathrm{~b} 05209$

117. Jurado-Sanchez B, Pacheco M, Rojo J, Escarpa A. Magnetocatalytic graphene quantum dots Janus micromotors for bacterial endotoxin detection. Angew Chem Int Edit. 2017;56 (24):6957-6961. doi:10.1002/anie.201701396

118. Wu J, Balasubramanian S, Kagan D, Manesh KM, Campuzano S, Wang J. Motion-based DNA detection using catalytic nanomotors. Nat Commun. 2010;1:36.

119. Wang Y, Zhou C, Wang W, et al. Photocatalytically powered matchlike nanomotor for light-guided active SERS sensing. Angew Chem Int Edit. 2018;57(40):13110-13113. doi:10.1002/ anie. 201807033

120. Gupta R, Rai B. In-silico design of nanoparticles for transdermal drug delivery application. Nanoscale. 2018;10(10):4940-4951. doi:10.1039/c7nr07898f
International Journal of Nanomedicine

\section{Publish your work in this journal}

The International Journal of Nanomedicine is an international, peerreviewed journal focusing on the application of nanotechnology in diagnostics, therapeutics, and drug delivery systems throughout the biomedical field. This journal is indexed on PubMed Central, MedLine, CAS, SciSearch ${ }^{\mathbb{R}}$, Current Contents ${ }^{\mathbb{B}} /$ Clinical Medicine,
Journal Citation Reports/Science Edition, EMBase, Scopus and the Elsevier Bibliographic databases. The manuscript management system is completely online and includes a very quick and fair peer-review system, which is all easy to use. Visit http://www.dovepress.com/ testimonials.php to read real quotes from published authors. 